IASSNS-HEP-95/8

\title{
Aharonov-Bohm Order Parameters for Non-Abelian Gauge Theories
}

\author{
Hoi-Kwong Lo \\ School of Natural Sciences, Institute for Advanced Study, Olden Lane, \\ Princeton, NJ 08540, U.S.A.
}

(October 17, 2018)

\begin{abstract}
The Aharonov-Bohm effect has been invoked to probe the phase structure of a gauge theory. Yet in the case of non-Abelian gauge theories, it proves difficult to formulate a general procedure that unambiguously specifies the realization of the gauge symmetry, e.g. the unbroken subgroup. In this paper, we propose a set of order parameters that will do the job. We articulate the fact that any useful Aharonov-Bohm experiment necessarily proceeds in two stages: calibration and measurement. World sheets of virtual cosmic string loops can wrap around test charges, thus changing their states relative to other charges in the universe. Consequently, repeated flux measurements with test charges will not necessarily agree. This was the main stumbling block to previous attempts to construct order parameters for non-Abelian gauge theories. In those works, the particles that one uses for calibration and subsequent measurement are stored in separate "boxes". By storing all test particles in the same "box", we show how quantum fluctuations can be overcome. The importance of gauge fixing is also emphasized.
\end{abstract}


PACS numbers:11.15.-q,03.65.Bz,03.80.+r

Typeset using REVTEX 


\section{INTRODUCTION}

A gauge theory can have an interesting phase diagram. Depending on it Higgs structure and on the parameters of the Higgs potential, the theory may be in a Coulomb phase, a Higgs phase or a confinement phase. Order parameters that distinguish among the various

phases have been proposed. Consider pure $S U(N)$ gauge theory. The Wilson loop operator

$$
W(C)=\operatorname{tr}\left[P \exp \left(i g \oint_{C} a \cdot d x\right)\right]
$$

may be regarded as an insertion of a classical source of charges, transforming as the defining representation of $S U(N)$, that propagates along the worldline $C$. In the confinement phase, $C$ is the boundary of the worldsheet of an electric flux tube. For large loops, $W(C)$ exhibits area-law behavior

$$
W(C) \sim \exp [-\kappa A(C)]
$$

where $A(C)$ is the minimal area of a surface bounded by $C$, and $\kappa$ is the string tension. In the Higgs phase, electric flux is screened, and the Wilson loop has the perimeter-law behavior

$$
W(C) \sim \exp [-\mu P(C)]
$$

where $P(C)$ is the length of $C$. Thus, $W(C)$ is a useful order parameter for pure $S U(N)$ gauge theory.

Once dynamical quarks (a matter field that transforms non-trivially under the center $Z_{N}$ of $S U(N))$ are introduced, the confining and Higgs phases can no longer be distinguished by the above criterion. Quark-antiquark pairs appear as quantum fluctuations, allowing electric flux tube to break. The Wilson loop therefore always obeys the perimeter law. In the case of $S U(N)$ discussed above, it is widely believed that no sharp boundary exists between the confinement phase and the Higgs phase of interest [1].

However, two types of Higgs phases are possible, depending on whether the center $Z_{N}$ is manifest or broken. If the $Z_{N}$ symmetry is manifest, there will be a $Z_{N}$ superselection rule. 
If it is unbroken, no such superselection rule exists. It is presumably the Higgs phase with spontaneous broken $Z_{N}$ that is indistinguishable from the confining phase.

How can we distinguish between the two types of Higgs phases? Topological defects are potentially useful. In this paper, we assume, for simplicity, that a gauge theory with a (simply-connected) symmetry group $K$ is spontaneously broken in two stages: first to a discrete subgroup $G$ at mass scale $v_{1}$, then to $H$, a subgroup of $G$ at mass scale $v_{2} \ll$ $v_{1}$. We will focus on the second stage of the symmetry breakdown and construct a set of order operators for our investigation. The first stage of symmetry breakdown gives rise to topological vortices (in $2+1$ dimensions) and cosmic strings (in $3+1$ dimensions). A cosmic string carries a "flux" which is labelled by an element of the unbroken group $G$. [Vortices are classified by the homotopy group $\pi_{1}(K / G)$ [2, 3]. It follows from the exact homotopy sequence $\cdots \rightarrow \pi_{1}(K) \rightarrow \pi_{1}(K / G) \rightarrow \pi_{0}(G) \rightarrow \pi_{0}(K)$ that $\pi_{1}(K / G) \simeq \pi_{0}(G) \simeq G$ under the assumptions that $K$ is simply-connected and $G$ is discrete. To be more precise, the spectrum of stable vortices only spans $G$ as a vortex associated with an element $g$ of $G$ may be unstable to the decay into two or more vortices of the same total flux. This is, however, of no interest to us.] Notice that there is a one-one correspondence between the elements of the unbroken group and the topological classes of stable string loops. This remains true even when $G$ is spontaneously broken into $H$, in which case the topological classes of stable string loops are labelled by elements of $H$. (An element, $a$, of $G$ which is not in $H$ is not associated with isolated string loops, but with string loops that are bounded to domain walls.) Therefore, by reading off the spectrum of stable strings, we can determine the unbroken group and decide whether the second stage of symmetry breakdown has occurred.

So, the question becomes: how can we read off the fluxes of the stable vortices in our theory? A string generally has long range Aharonov-Bohm [4] type interactions with various particles: the wave function of a particle acquires a non-Abelian phase when it is covariantly transported around a string [5, [5].

This simple phenomenon has deep consequences. Since the Aharonov-Bohm interaction is long range and no local operator can destroy an object with an infinite-range interaction, 
gauge theories with such interactions obey non-trivial superselection rules. The structure of the superselection sector can be invoked to distinguish among the various possible phases of a gauge theory. Moreover, the Aharonov-Bohm interaction exposes the limitations of the classical no-hair conjecture in black hole physics. A black hole may carry quantum numbers that are detectable only by means of quantum mechanical interference experiments with cosmic strings [7]. By sending particles around the various string loops and measuring the non-Abelian Aharonov-Bohm factors acquired by them, we can read off the spectrum of stable strings. The usage of the Aharonov-Bohm effect between charged particles and cosmic strings to probe the unbroken group of a non-Abelian gauge theory was proposed by Preskill and Krauss [6]. It requires a framework that takes full account of the effect of virtual particles and virtual string loops. Nevertheless, generalization to non-Abelian gauge theories turned out to be very elusive. In spite of much progress in our understanding of the subtler aspects of non-Abelian gauge theories [8 [12] it proves difficult [13 15] to formulate a general procedure that unambiguously specifies the realization of the gauge symmetry.

In this paper, we construct a set of order parameters that will do the job, elaborating on our key results stated in Ref. [16]. These order parameters are closely related to some operators investigated by Alford et al. three years ago [15]. They, however, immediately rejected their operators because, in their original forumlation, they were plagued by quantum fluctuations.

As we will see below, any useful Aharonov-Bohm experiment to determine the flux of a string loop necessarily proceeds in two stages: calibration and subsequent measurement. Both stages involve interference experiments with two beams of charged particles one of which traverses the string loop while the other just sits at the base point. To construct an order parameter for non-Abelian gauge theories, the effects of virtual string loops need to be considered. The formulation by Alford et al. corresponded to storing the charged particles that one uses for calibration and those for measurement in separate boxes. (See FIG. 1a.) It was only because of this decoupling of the two that quantum fluctuations due to virtual string loops may spoil the result. What happen is that a small world sheet of 
virtual string loop can wind around the box which contains all the calibrating particles, changing their state relative to those we used for subsequent measurement (that are stored in another box). As a result, the subsequent flux measurement gives an answer different from the calibrated value. If we keep both types of particles in the same box (and for the subsequent measurement, an interference experiment is performed between a wavepacket that is kept in the box and another that is parallel transported around the string of interest) (See FIG. 1b.), we argue that the effect of virtual strings can be safely ignored.

Another important issue that has been overlooked in previous work 14, 15, is gauge fixing. Recall that we are interested in studying the symmetry breakdown of $G$ into a subgroup $H$. Without gauge fixing, an element $h$ in an unbroken group $H$ has no invariant meaning. Under a global gauge transformation by $g \in G, h \rightarrow g h g^{-1}$. However, after symmetry breakdown strings with fluxes $h$ and $g h g^{-1}$ are generally not gauge invariant. To test whether a symmetry breaking has occurred, one has to choose a field $\phi$ as a candidate for the Higgs field, gauge fix $\phi=\phi_{0}$ and consider $H\left(\phi_{0}\right)$ and its conjugacy classes and representations. By dealing with the issues of quantum fluctuations and gauge fixing squarely, we see clearly how a gauge group $G$ is reduced to an effective subgroup $H$ at low energies. Subsequent symmetry breaking of $H$ can be studied in a similar manner.

We emphasize that the idea of using the Aharonov-Bohm effect to probe the phase diagram is rather general. In particular, the existence of stable cosmic strings is not a necessary condition. Nor is the symmetry group required to be discrete. (After all, the Aharonov-Bohm effect exists in ordinary QED which has a continuous symmetry.) One can always imagine setting up localized magnetic flux tubes and studying their long ranged Aharonov-Bohm interactions with charged particles. Suppose symmetry breaking now occurs. Some of those flux tubes will then become the boundaries of unstable domain walls and the Aharonov-Bohm interactions will be modified in such a way to be consistent with the symmetry of the unbroken group. It is only for simplicity that we restrict our discussion to gauge theories with discrete symmetry only. Besides, we sometimes make use of non-gauge invariant operators. The reader should, however, bear in mind that the whole discussion 
can be recast in objects that are gauge-invariant with respect to the unbroken low energy symmetry group.

The organization of the paper is as follows. Section 2 concerns the relation between Dirac quantization condition and the Aharonov-Bohm effect. In Section 3, we discuss the basic ideas behind the construction of the Aharonov-Bohm order parameters. How quantum fluctuations can change the state of test particles and affect the result of a subsequent Aharonov-Bohm experiment is the main subject of our study in Section 4. Sections 5 and 6 concern respectively quantum fluctuations and gauge fixing, two crucial issues in the construction. Finally, we present our discussions and conclusions in Section 7. In the Appendix, we review the application of the order parameters to $Z_{2}$ gauge-Higgs system by Preskill and Krauss.

\section{DIRAC QUANTIZATION CONDITION AND THE AHARONOV-BOHM EFFECT}

In his seminal paper of 1931, Dirac [17] proposed that the quantization of electric charge can be "explained" by postulating the existence of isolated magnetic poles. Specifically, he showed that, for a charged particle of charge $e$ moving in the field of a magnetic monopole of strength $\mu_{0}$, the consistency of its quantum mechanics demands that the product of the two charges satifies $e \mu_{0}=\frac{1}{2} n \hbar c$. This is the well-known Dirac quantization condition. Note that it has an explicit dependence on Planck's constant, and therefore on the quantum theory. Moreover, it shows a perfect symmetry between electricity and magnetism.

Generalization of this simple condition has deep implications. For instance, consider an underlying gauge theory with gauge group $G$ which is spontaneously broken into a subgroup $S U(N) / Z_{N}$. This theory admits magnetic monopoles with $Z_{N}$ magnetic charges. Fields that transform non-trivially under $Z_{N}$ and the monopole with minimal magnetic charge taken together do not satisfy the Dirac condition. One immediately sees that the quantum

mechanics of a system of a free minimal monopole and a free $Z_{N}$ charge is inconsistent. 
Thus, one is led to the conclusion that either the minimal monopole or the $Z_{N}$ charge must be confined. This is the basic concept behind t' Hooft's discussion on quark confinement [1]. The t' Hooft operator $B_{n}\left(C^{\prime}\right)$ essentially introduces, as a classical source, a $Z_{N}$ monopole worldline along the curve $C^{\prime}$. It is the magnetic analogue of the Wilson loop operator $A^{\nu}(C)$, which introduces, as a classical source, a worldline of a charged particle in the representation $\nu$. t' Hooft considered a Green's function $\left\langle B_{n}\left(C^{\prime}\right) A^{\nu}(C)\right\rangle$. If the Dirac quantization condition is not satisfied, this object is generally multivalued.

If the charged particle is regarded as free, it will see the Dirac string associated with the monopole. That is to say that the Dirac string is physical and it has a long-range AharonovBohm interaction with the charged particle. The Green's function can be made single-valued if a world sheet $\Sigma^{*}$ of the Dirac string is chosen. i.e. $\left\langle B_{n}\left(C^{\prime}, \Sigma^{*}\right) A^{\nu}(C)\right\rangle$ is single-valued. If $C^{\prime}$ shrinks to a point, we replace the operator $B_{n}\left(C^{\prime}, \Sigma^{*}\right)$ by $F\left(\Sigma^{*}\right)$ which introduces a closed world sheet of string. We emphasize that the Aharonov-Bohm effect is quantum mechanical. It can be determined only through interference experiments. (Incidentally, the Aharonov-Bohm interactions between magnetic monopole and electric flux tubes can be discussed in a totally analogous manner [15]: If a monopole is regarded as free, (i.e. there is no physical Dirac string) charged particles which do not satisfy the Dirac quantization condition are confined. Therefore, it is appropriate to consider operators $G^{\nu}(\Sigma)$ and $B_{n}$ which respectively introduce a world sheet of an electric flux tube and a worldline of a magnetic monopole.) As we will see in next section, the operators $F$ and $A$ will play a key role in the construction of order parameters for gauge theories.

\section{ORDER PARAMETERS}

In this section, we consider the order parameters for Abelian gauge theories proposed by Preskill and Krauss [6] and elaborated by Alford et al. [15]. By the well-known Elitzur's theorem [18], the Higgs field $\phi$ is not a true order parameter because it has no gauge invariant

meaning. The order parameters proposed by Preskill and Krauss make essential use of the 
Aharonov-Bohm interactions between cosmic strings and charged particles.

While our ideas are general, for simplicity, we shall discuss the concepts for only the case of finite gauge groups [6, 14, [15]. More specifically, we consider a discrete gauge group $G$ which arises as a result of a symmetry breaking of a simply-connected group $K$. We are interested in investigating the further symmetry breakdown of $G$ into a subgroup $H$ at low energies.

The symmetry breakdown of $K$ into $G$ leads to the existence of stable vortices labelled by elements of $G$. (More details will be discussed in the next Section.) Suppose we prepare (and calibrate) a set of cosmic strings. In a free $G$ charge phase, a particle scattering off a cosmic string will recover the flux of the string as a non-Abelian Aharonov-Bohm factor. If $G$ is broken into $H$ instead, then the elements of $G$ that are not in $H$ are not associated with isolated cosmic strings, but with strings that are boundaries of domain walls. Such domain walls are unstable and will decay via spontaneous nucleation of string loops [2], 3]. Consider a string of flux $a \notin H$ bounded by a domain wall. Many holes eventually appear in the wall bounded to $a$. They collide with one another. Ultimately, the one with the least string tension, $b$, will dominate the decay. As a result of this decay, the $a$ string is turned into a composite string with total flux $a b^{-1} \in H$. Hence, a particle scattering off a cosmic string in a free $H$ charge phase will only acquire phases that are associated with the elements of $H$. [We must include the possibility that the set of strings with the least string tension has more than one elements. For instance, a string with the least string tension can have a flux $b$ or $b^{\prime}$. Therefore, the Aharonov-Bohm experiment may show that the composite flux after the decay of the domain wall is $a b$ or $a b^{\prime}$.]

The basic idea of the Aharonov-Bohm order parameters is the following. Since, in the case of discrete gauge theories, there is always a one-one correspondence between the topological classes of stable vortices and the elements of the unbroken group (see the Introduction and the next Section for details), we can figure out the manifest symmetry group just by reading out the spectrum of stable vortices. To read off the spectrum, we use the Aharonov-Bohm effect. Physically, we proceed in two stages: 1) Prepare (Calibrate) a localized vortex for each 
element of G. 2) Send particles around those vortices to measure the associated AharonovBohm factors. The results of the measurements can tell us whether the symmetry breaking from $G$ to $H$ has occurred or not.

Let us consider the first stage of an Aharonov-Bohm experiment: Calibration of string flux [19]. We need an operator which inserts, as a classical source, a string world sheet of flux $a$. It was suggested in [5] that when a $U(1)$ gauge symmetry is spontaneously broken into $Z_{N}$, the discrete $Z_{N}$ charge $Q_{\Sigma^{*}}$ contained in a closed surface $\Sigma^{*}$ can still be measured via the Gauss law:

$$
F\left(\Sigma^{*}\right)=\exp \left(\frac{2 \pi i}{N} Q_{\Sigma^{*}}\right)=\exp \left(\frac{2 \pi i}{N e} \int_{\Sigma^{*}} E \cdot d s\right) .
$$

$\left[F\left(\Sigma^{*}\right)\right.$ is closely related to the 't Hooft loop operator [1]. Strictly speaking, Eq. (4) is incorrect as an operator statement. Charge can be defined in two different ways: the Coulomb charge and the Aharonov-Bohm charge. The Coulomb charge is the one that enters in the Coulomb force between two charged particles. It is screened in a Higgs phase. Therefore, the Gauss law actually gives zero for the Coulomb charge. However, the Aharonov-Bohm interactions between confined flux tubes and charges are unscreened (See, for example, [20]). In Eq. (田), we are just making the heuristic statement that charges can be detected by their Aharonov-Bohm interactions with vortices.]

Now we turn to the operator which introduces classical charges into the system. An obvious choice would be the Wilson loop operator $W^{\nu}(C)$ where $\nu$ is an irreducible representation of the gauge group, $G$. Therefore, one might naively expect $F\left(\Sigma^{*}\right) W^{\nu}(C)$ to be the order parameter. This is not quite correct because quantum mechanical fluctuations near the surface $\Sigma^{*}$ cause an area law decay of the modulus of $F\left(\Sigma^{*}\right) \sim \exp \left(-\kappa A\left(\Sigma^{*}\right)\right)$. Fortunately, the phase of $F\left(\Sigma^{*}\right)$ remains unscreened and we can isolate it by dividing out its vacuum expectation value and obtain $\frac{F\left(\Sigma^{*}\right)}{\left\langle F\left(\Sigma^{*}\right)\right\rangle}$ [6]. Similarly, quantum fluctuations also lead to the exponential decay of the expectation value of $W(C)$. Therefore, the true order parameter for Abelian gauge theories is 6

$$
A^{\nu}\left(\Sigma^{*}, C\right)=\frac{F\left(\Sigma^{*}\right) W^{\nu}(C)}{\left\langle F\left(\Sigma^{*}\right)\right\rangle\left\langle W^{\nu}(C)\right\rangle} .
$$


In the free $Z_{N}$ charge phase, the order parameter (for the fundamental representation) gives

$$
\lim \left\langle A\left(\Sigma^{*}, C\right)\right\rangle=\exp \left(\frac{2 \pi i}{N} k\left(\Sigma^{*}, C\right)\right) .
$$

Here the order parameter takes a value that is independent of the details of $\Sigma^{*}$ and $C$ so long as the limit is taken with $\Sigma^{*}$ and $C$ increasing to infinite size, and with the closest approach of $\Sigma^{*}$ to $C$ also approaching infinity; $k\left(\Sigma^{*}, C\right)$ denotes the linking number of the surface $\Sigma^{*}$ and the loop $C$. ( $\Sigma^{*}$ and $C$ have to be far away from each other because we are only interested in the long range Aharonov-Bohm interactions.) On the other hand, if there are no free $Z_{N}$ charges, then we have

$$
\lim \left\langle A\left(\Sigma^{*}, C\right)\right\rangle=1 .
$$

The non-analytical behavior of $A\left(\Sigma^{*}, C\right)$ guarantees that the two phases are separated by a well-defined phase boundary. An order parameter can be easily generalized to probe the realisation of any Abelian discrete gauge symmetry.

\section{QUANTUM FLUCTUATIONS}

The case of non-Abelian gauge theories is more subtle. In this Section, we will show how quantum fluctuations can affect the result of a non-Abelian Aharonov-Bohm experiment, thus making the construction of the order parameter much more difficult.

Consider, in two spatial dimensions, a simply-connected gauge group $K$ which gets spontaneously broken into a discrete, non-Abelian subgroup $G$. This pattern of symmetry breaking will admit stable classical vortex solutions. Since the size of the vortex core is of the order of the inverse symmetry breaking scale, it is almost pointlike at low energies. We shall, therefore, ignore the core of the vortex and idealize it as a point singularity. The "flux" carried by a vortex is labelled by an element of the unbroken group $G$. To assign a group element to the vortex, we arbitrarily choose a "base point" $x_{0}$ and a path $C$, beginning and ending at $x_{0}$, that winds around the vortex. The effect of parallel transport in the gauge potential of the vortex is then encoded in the untraced Wilson loop operator [21.22] 


$$
U^{\nu}\left(C, x_{0}\right)=P \exp \left(i \int_{C, x_{0}} A\right)
$$

where $P$ denotes path ordering. Suppose a particle is in the initial state $|u\rangle$. When it winds around a vortex (or a cosmic string loop in three dimensions), its state becomes $U^{\nu}\left(C, x_{0}\right)|u\rangle$. The matrix $U^{\nu}\left(C, x_{0}\right)$ specifies a group element in the subgroup $G\left(x_{0}\right)$ of $K$ that preserves the Higgs condensate at the point $x_{0}$, since transport of the condensate around the vortex must return it to its original value. If $G$ is discrete, then the element assigned will remain unchanged as the path $C$ is smoothly deformed, as long as the path never crosses the cores of any vortices. (The gauge connection is locally flat outside the vortex cores, with curvature singularities at the cores.)

In the non-Abelian case, the flux, $a$, of a vortex is not a gauge invariant quantity. Upon a global gauge transformation by $g, a \rightarrow g a g^{-1}$. One might naively identify two vortices in the same conjugacy class as identical. However, this is not quite correct because there is only one overall global gauge degree of freedom. For example, if there are two vortices of flux $a$ and $b$, upon a global gauge transformation by $g$, we have $a \rightarrow g a g^{-1}, b \rightarrow g b g^{-1}$ [6, 12,22,23]. It is important to note that, two vortices with conjugate but different fluxes (in some gauge) are not identical to each other. Consider two vortices of conjugate but different fluxes, $a$ and $b=c a c^{-1}$ in some gauge. They are clearly different in this gauge. (As we will see below, a particle that winds around the $a$ vortex first and then in the inverse sense around the $b=c a c^{-1}$ vortex will pick up the Aharonov-Bohm factor depending on $c a^{-1} c^{-1} a$. Here our convention for multiplication is from right to left. If these two vortices had the same flux, the Aharonov-Bohm factor would be trivial instead.) Now under a global gauge transformation, by $g, a \rightarrow g a g^{-1}$ and $c a c^{-1} \rightarrow g c a c^{-1} g^{-1}$ and the two fluxes clearly remain different. Notice also that the one-one correspondence mentioned in the last Section is between the spectrum of stable vortices and the elements of the unbroken group rather than its conjugacy classes. It is, therefore, crucial to be able to distinguish between vortices that are associated with different elements in the same conjugacy class. To obtain this one-one correspondence, one has to fix a gauge. Fortunately, a change of gauge merely amounts to a change of basis and 
the one-one correspondence still exists in the new gauge. The analogy with isospin may be helpful for understanding why vortices of conjugate but different fluxes should be regarded as non-identical. In an isospin symmetric universe, it is a matter of convention to call an object a neutron rather than a proton. However, once we call an object a neutron, our convention has been fixed and we will be able to distinguish a proton from a neutron by comparing their isospins.

If a particle that transforms as an irreducible representation $(\nu)$ traverses a string of flux $a$, the Aharonov-Bohm phase that it acquires, when averaged over a basis for the representation, is

$$
\frac{1}{n_{\nu}} \chi^{\nu}(a)
$$

where $\chi^{\nu}$ and $n_{\nu}$ are the character and the dimension of $(\nu)$. Now if two strings of fluxes $a$ and $b$ are patched incoherently, the Aharonov-Bohm phase acquired by the particle that travels around the two strings in succession is

$$
\frac{1}{n_{\nu}} \chi^{\nu}(a) \frac{1}{n_{\nu}} \chi^{\nu}(b)
$$

If the two strings are combined coherently instead, the phase acquired is

$$
\frac{1}{n_{\nu}} \chi^{\nu}(a b)
$$

Thus, the Aharonov-Bohm factor associated with a coherent pair of string is not just the product of the Aharonov-Bohm factors associated with the two individual strings. This coherence property is the hallmark of the non-Abelian Aharonov-Bohm effect.

The above discussion ignores the effect of quantum fluctuations. Quantum fluctuations can spoil the coherence between various strings. It is instructive to consider a double slit experiment with two vortices of fluxes $g_{1}$ and $g_{2}$ placed between the two slits and using a particle-antiparticle pair in the representation $(\nu)$. Let us put the $g_{2}$ vortex in front of the two slits and the $g_{1}$ vortex behind. Suppose the particle-antiparticle pair is initially in the zero-charged state, i.e., 


$$
|0, \nu\rangle=\frac{1}{\sqrt{n}_{\nu}} \sum_{i}\left|e_{i}^{\nu} \otimes e_{i}^{* \nu}\right\rangle
$$

Let us first consider the case without quantum fluctuations. When the particle traverses the double slit, the state of the entire state will transform in the following manner:

$$
\left|g_{1}, g_{2}\right\rangle \otimes \frac{1}{\sqrt{n}_{\nu}} \sum_{i}\left|e_{i}^{\nu} \otimes e_{i}^{* \nu}\right\rangle \rightarrow\left|g_{1}, g_{2}\right\rangle \otimes \frac{1}{\sqrt{n}_{\nu}} \sum_{i j}\left|e_{j}^{\nu} \otimes e_{i}^{* \nu}\right\rangle D_{j i}^{(\nu)}\left(g_{1} g_{2}\right)
$$

where $D_{j i}^{(\nu)}$ are the matrix elements of the representation $(\nu)$.

For the particle-antiparticle pair to annihilate, we project onto the zero-charged state using the projection operator

$$
P_{\text {particle }}=|0, \nu\rangle\langle 0, \nu|
$$

to obtain

$$
\begin{aligned}
& \left|g_{1}, g_{2}\right\rangle \otimes \frac{1}{\sqrt{n}} \sum_{\nu}\left|e_{k}^{\nu} \otimes e_{k}^{* \nu}\right\rangle \sum_{i j k^{\prime}}\left\langle e_{k^{\prime}}^{\nu} \otimes e_{k^{\prime}}^{* \nu} \mid e_{j}^{\nu} \otimes e_{i}^{* \nu}\right\rangle \frac{1}{n_{\nu}} D_{j i}^{(\nu)}\left(g_{1} g_{2}\right) \\
\rightarrow & \left|g_{1}, g_{2}\right\rangle \otimes|0, \nu\rangle \frac{1}{n_{\nu}} \chi^{\nu}\left(g_{1} g_{2}\right) .
\end{aligned}
$$

In other words, in the absence of virtual processes, the interference pattern will determine the Wilson loop to be $\frac{1}{n_{\nu}} \chi^{\nu}\left(g_{1} g_{2}\right)$.

Let us now turn to quantum fluctuations. Notice that two beams of particles are split and recombined in an Aharonov-Bohm experiment. For those quantum fluctuations (such as virtual vortices that wind around only one of the two beams) whose effects do not depend on the flux of vortex with which we are performing the Aharonov-Bohm interference experiment, their effect can simply be factored out. (Cf. Eq. (司).) However, there are quantum fluctuations (that affect both beams) whose effects cannot be factored out: consider the spontaneous nucleation of a charge-zero virtual vortex-antivortex pair in the conjugacy class $\left[g^{\prime}\right]$, i.e.,

$$
\left|0,\left[g^{\prime}\right]\right\rangle=\frac{1}{\sqrt{n}_{\left[g^{\prime}\right]}} \sum_{g \in\left[g^{\prime}\right]}\left|g, g^{-1}\right\rangle
$$


(here $n_{\left[g^{\prime}\right]}$ is the number of elements in the conjugacy class $\left[g^{\prime}\right]$ ) in the region between the two slits and the $g_{1}$ vortex. Suppose the vortex and antivortex move apart just before the double slit experiment. When a particle traverses the two slits, the state of the entire system will change as follows:

$$
\begin{aligned}
& \left|g_{1}, g_{2}\right\rangle \otimes \frac{1}{\sqrt{n}_{\left[g^{\prime}\right]}} \sum_{g \in\left[g^{\prime}\right]}\left|g, g^{-1}\right\rangle \otimes \frac{1}{\sqrt{n}} \sum_{i}\left|e_{i}^{\nu} \otimes e_{i}^{* \nu}\right\rangle \\
\rightarrow & \left|g_{1}, g_{2}\right\rangle \otimes \frac{1}{\sqrt{n_{\left[g^{\prime}\right]}}} \frac{1}{\sqrt{n}_{\nu}} \sum_{g \in\left[g^{\prime}\right]} \sum_{i j}\left|g, g^{-1}, e_{j}^{\nu} \otimes e_{i}^{* \nu}\right\rangle D_{j i}^{(\nu)}\left(g g_{1} g^{-1} g_{2}\right) .
\end{aligned}
$$

(i.e., for each $g \in\left[g^{\prime}\right]$, the particle "sees" a flux $g g_{1} g^{-1} g_{2}$ rather than $g_{1} g_{2}$.) Now to make sure that the virtual vortex-antivortex pair will annihilate, we apply the projection operator

$$
P_{\text {vortex }}=\left|0,\left[g^{\prime}\right]\right\rangle\left\langle 0,\left[g^{\prime}\right]\right|
$$

We also project onto the zero-charged state for the particle-antiparticle pair by using the operator $P_{\text {particle }}$ defined in Eq. (14). Therefore, we obtain

$$
\begin{aligned}
\left|g_{1}, g_{2}\right\rangle & \otimes \frac{1}{\sqrt{n}_{\left[g^{\prime}\right]}} \frac{1}{\sqrt{n}} \sum_{\nu \in\left[g^{\prime}\right]} \sum_{i j}\left|g, g^{-1}, e_{j}^{\nu} \otimes e_{i}^{* \nu}\right\rangle D_{j i}^{(\nu)}\left(g g_{1} g^{-1} g_{2}\right) \\
\stackrel{P_{\text {vortex }} P_{\text {particle }}}{\longrightarrow}\left|g_{1}, g_{2}\right\rangle & \otimes\left|0,\left[g^{\prime}\right]\right\rangle|0, \nu\rangle \frac{1}{n_{\left[g^{\prime}\right]}} \sum_{g \in\left[g^{\prime}\right]} \chi^{\nu}\left(g g_{1} g^{-1} g_{2}\right) .
\end{aligned}
$$

Notice that the Aharonov-Bohm factor acquired by the particle,

$$
\frac{1}{n_{\left[g^{\prime}\right]}} \sum_{g \in\left[g^{\prime}\right]} \chi^{\nu}\left(g g_{1} g^{-1} g_{2}\right)
$$

is generally different from the corresponding Aharonov-Bohm factor without the virtual vortex pair $\left(\frac{1}{n_{\nu}} \chi^{\nu}\left(g_{1} g_{2}\right)\right)$. This result shows clearly that quantum fluctuations due to virtual string loops can spoil the hallmark of the non-Abelian Aharonov-Bohm effect- - the coherence between two strings in a double slit measurement [24]. This tends to create great difficulty in interpreting the outcome of a non-Abelian Aharonov-Bohm experiment. We will come back to discuss how this difficulty can be overcome in Section 5 .

A somewhat simpler but less precise way of stating our result is that for non-Abelian gauge theories repeated Aharonov-Bohm flux measurements do not necessarily agree 15 . 
Suppose we send out some of the charged particles to calibrate a vortex and keep the rest elsewhere for later use. A virtual vortex-antivortex pair is spontaneously nucleated and the virtual vortex winds around the charged particles that we use for calibration. We now send out the remaining charged particles to measure the flux of the vortex again. The claim is that we will find that its flux has been conjugated. As shown in the spacetime diagram (FIG. 2), the virtual vortex worldline has a non-trivial linking number with the union of the following three objects: the worldline of the vortex under consideration and the worldlines of the charged particles that we use for the calibration AND that of the ones that we use for subsequent measurement. The topological linking number shows that the virtual vortex worldline conjugates the result of the subsequent measurement relative to the calibrated value [24].

We remark that there are other kinds of quantum fluctuations in which quantum numbers are exchanged between two objects. For instance, an $i$ vortex can momentarily emit a -1 vortex, turning itself into a $-i$ vortex. If this -1 vortex is absorbed by the $j$ vortex, quantum numbers will be exchanged between the $i$ and $j$ vortices. However, these types of quantum fluctuations are uninteresting for our purposes and will not be considered any further in this paper.

\section{ORDER PARAMETERS FOR NON-ABELIAN GAUGE THEORIES}

\section{A. General Formulation}

We have seen in Section 3 how the Aharonov-Bohm effect can be used to probe the phase diagram of an Abelian gauge theory. In this section, we would like to extend the construction to non-Abelian gauge theories. This generalization turns out to be difficult and all previous attempts have not been entirely successful [14,15]. For ease of discussion, let us fix the gauge

completely. As discussed in Section 4, we shall regard vortices of conjugate but different fluxes to be non-identical. For each element $a \in G$, we need to define an operator $F_{a}\left(\Sigma^{*}\right)$ 
which introduces a world sheet $\Sigma^{*}$ of a string of flux $a$. (The fact that the flux, $a$, is not a gauge invariant quantity is not a problem because, at the end of our discussion, we will apply the overall global gauge degree of freedom to obtain gauge-invariant operators.)

For simplicity, consider the case of $2+1$ dimensions. How can we specify the worldline $\Sigma^{*}$ of a vortex of flux $a$ using the operator $F_{a}\left(\Sigma^{*}\right)$ ? One can imagine assembling a laboratory of test particles at some arbitrary base point $x_{0}$ and choosing a basis for various representations there. We then send two beams of particles to pass on either side of the vortex, recombine the beams and study the resultant interference pattern. In fact, a sequence of the AharonovBohm experiments has to be performed over time to specify the whole worldline of the vortex. To localized the vortex worldline to (be close to) $\Sigma^{*}$, those calibration experiments have to be done near to the vortex. (To be more precise, for each of those calibration experiments, the two beams involved are split only in a small region near $\Sigma^{*}$. One of the beam then traverses the vortex and the two beams are recombined immediately afterwards.) The case of $3+1$ dimensions is the same except that now we have to specify the worldsheet of a string.

In a cubic four-dimensional lattice formulation, it is convenient to put a string world sheet on a closed surface $\Sigma^{*}$ on the dual lattice. Let $\Sigma$ be the set of plaquettes threaded by $\Sigma^{*}$. Here comes the crucial point. We pick our calibration paths: for each plaquette $P$ in $\Sigma$, we choose a path, $l_{P}$, that runs from the base point $x_{0}$ to a corner of the plaquette [15]. Calibration of the plaquette is done along the path $l_{P} P l_{P}^{-1}$. The operator $F_{a}\left(\Sigma^{*}, x_{0},\left\{l_{P}\right\}\right)$ inserts, as a classical source, a string world sheet $\Sigma^{*}$ calibrated along paths $\left\{l_{P}\right\}$ and modifies the gauge action in the following manner. Suppose that the Euclidean plaquette action is

$$
S_{\text {gauge }, P}^{(R)}=-\beta \chi^{(R)}\left(U_{P}\right)+c . c .
$$

where $R$ is some representation of the gauge group that defines the theory and $U_{P}=\prod_{l \in P} U_{l}$ associates with each plaquette (labelled by $\mathrm{P}$ ) the ordered product of the four $U_{l}$ 's associated with the oriented links of the plaquette. The insertion of $F_{a}\left(\Sigma^{*}, x_{0},\left\{l_{P}\right\}\right)$ modifies the action on each plaquette in $\Sigma$ to

$$
S_{\text {gauge }, P}^{(R)} \rightarrow-\beta \chi^{(R)}\left(V_{l_{P}} a V_{l_{P}}^{-1} U_{P}\right)+c . c .
$$


where

$$
V_{l_{P}}=\prod_{l \in l_{P}} U_{l}
$$

(This procedure can be generalized to insert coherently many string loops using an operator $F_{a_{1}, a_{2}, \cdots, a_{n}}\left(\Sigma_{1}^{*}, \Sigma_{2}^{*}, \cdots, \Sigma_{n}^{*}, x_{0},\left\{l_{P}\right\}\right)$. See below. Note that for coherent insertion, it is crucial to choose the same base point $x_{0}$ for all string loops. This operator is not gauge invariant. Upon gauge transformation by $g$ at the base point, it changes to $\left.F_{g a_{1} g^{-1}, g a_{2} g^{-1}, \cdots, g a_{n} g^{-1}}\left(\Sigma_{1}^{*}, \Sigma_{2}^{*}, \cdots, \Sigma_{n}^{*}, x_{0},\left\{l_{P}\right\}\right).\right)$

Up to now, we have been vague about the choice of the "tails" $\left\{l_{P}\right\}$ (i.e. the paths for the calibration). As it turns out, the choice is actually quite important. Unless the tails $\left\{l_{P}\right\}$ are chosen in a judicious manner, because of quantum fluctuations, there is no guarantee that a subsequent measurement of the flux of a calibrated string will recover the same result.

Now we turn to the operator which introduces classical charges into the system. After gauge fixing, all information of the non-Abelian Aharonov-Bohm effect is encoded in the untraced Wilson loop operator

$$
U^{(\nu)}\left(C, x_{0}\right)=D^{(\nu)}\left(\prod_{l \in C} U_{l}\right)
$$

where $C$ is a closed loop around $x_{0}$ and $\nu$ is an irreducible representation of the gauge group $G$. Conceptually, after gauge fixing all the matrix elements in $U^{(\nu)}\left(C, x_{0}\right)$ can, in principle, be determined by interfering charged particles in the representation $\nu$ that traverse $C$ with those that stay at the base point [19]. Just like $F_{a}$, the operator $U^{(\nu)}\left(C, x_{0}\right)$ is not gauge invariant.

When $\beta \gg 1$, the plaquettes are hard to excite. Therefore, configurations with only a small number of frustrated plaquettes will be important and we can expand in powers of $\exp (-\beta)$ (or equivalently in terms of world sheets of virtual string loops). When $F_{a}\left(\Sigma^{*}, x_{0},\left\{l_{P}\right\}\right)$ is inserted, the configuration at weak couplings that has none of its plaquette frustrated is an "a-forest" [14] in which, roughly speaking, all the links that intersect the minimal surface with boundary $\Sigma^{*}$ are of flux $a$. (FIG. 3.) Suppose the Wilson loop links 
once with the string loop. Exactly one of its links, $l$ (say with a flux $V_{l}$ ), is an $a$-forest link and this gives a flux $a$ for the Wilson loop. More generally, in a phase with free $G$ charges, and in the leading order of weak coupling perturbation theory, the operator [15]

$$
\begin{aligned}
\left\langle A_{a}^{\nu}\left(\Sigma^{*}, x_{0},\left\{l_{P}\right\} ; C\right)\right\rangle & \left.=\frac{\left\langle F_{a}\left(\Sigma^{*}, x_{0},\left\{l_{P}\right\}\right) U^{(\nu)}\left(C, x_{0}\right)\right\rangle}{\left\langle F_{a}\left(\Sigma^{*}, x_{0},\left\{l_{P}\right\}\right)\right\rangle\left\langle\operatorname{tr} U^{(\nu)}\left(C, x_{0}\right)\right.}\right\rangle \\
& =\frac{1}{n_{\nu}} D^{\nu}\left(a^{k\left(\Sigma^{*}, C\right)}\right)
\end{aligned}
$$

where $k\left(\Sigma^{*}, C\right)$ is the linking number of the surface $\Sigma^{*}$ and the loop $C$ and the limit that $\Sigma^{*}$ and $C$ are infinitely large and far away is taken. This shows that, once a string loop is calibrated to be of flux $a$ along the paths $\left\{l_{P}\right\}$, a subsequent interference experiment with a charged particle will recover the same non-Abelian Aharonov-Bohm factor.

\section{B. Quantum Fluctuations}

However, owing to quantum fluctuations, higher order terms in the weak coupling expansion may spoil this result [15]. Recall that the dominant contribution in a weak coupling expansion comes from configurations with a low density of frustrated plaquettes (or equivalently, a low density of virtual string loops). Now in the definition of $F_{a}\left(\Sigma^{*}, x_{0},\left\{l_{P}\right\}\right)$, for each plaquette $P$, there is a long tail of links $l_{P}$ that connects it to $x_{0}$. This is the calibration path for that particular plaquette. Alford et al. have considered the choice in which the long tails, $\left\{l_{P}\right\}$, from all the plaquettes finally merge together and connect to the base point through a single link which is not on the Wilson loop. Essentially, they keep all the charged particles for the calibration in a small box whose worldline runs from $x_{0}$ to $y_{0}$ before performing the experiment. (See FIG. 4.) However, this choice is vulnerable to quantum fluctuations. Totally analogous to our discussion concerning FIG. 2 in Section 4, consider the spontaneous nucleation of a virtual vortex-antivortex pair whose worldline is non-trivially linked to the union of three object: the Wilson loop, the tails and the string loop under calibration. This will conjugate the measured flux relative to the calibrated value. In the weak coupling expansion, such a configuration has a single excited link on the 
path that connects $x_{0}$ to $\Sigma^{*}$. This causes (in three spacetime dimensions) the excitation of four plaquettes and is suppressed by terms that are independent of the size of $\Sigma^{*}$ and $C$ or the separation between them. Thus, higher order corrections render the flux uncertain up to conjugation and this operator is useless as an order parameter. This was the conclusion drawn by Alford et al. [15].

Such a conclusion is unwarranted as it is based on an implicit choice of $\left\{l_{P}\right\}$. Before we present our resolution, let us note another related problem that we have already raised in the last Section. It is a subtle issue to maintain the coherence between various strings when quantum fluctuations are taken into account. With this implicit choice of long tails, these quantum fluctuations do destroy such coherence. This is because the calibrated flux of one string may be conjugated while the elements associated with others are unaffected. This relative change in flux is highly physical and does not go away even when we take the trace of our operator.

Consider the operator

$$
\operatorname{tr} A_{a_{1}, a_{2}, \cdots, a_{n}}^{\nu}\left(\Sigma_{1}^{*}, \Sigma_{2}^{*}, \cdots, \Sigma_{n}^{*}, x_{0},\left\{l_{P}\right\} ; C\right)=\frac{F_{a_{1}, a_{2}, \cdots, a_{n}}\left(\Sigma_{1}^{*}, \Sigma_{2}^{*}, \cdots, \Sigma_{n}^{*}, x_{0},\left\{l_{P}\right\}\right) W^{\nu}(C)}{\left\langle F_{a_{1}, a_{2}, \cdots, a_{n}}\left(\Sigma_{1}^{*}, \Sigma_{2}^{*}, \cdots, \Sigma_{n}^{*}, x_{0},\left\{l_{P}\right\}\right)\right\rangle\left\langle W^{\nu}(C)\right\rangle}
$$

It was suggested in Ref. [15] that when a charged particle winds around vortices of flux $a_{n}, a_{n-1}, \cdots, a_{2}, a_{1}$ in succession, it will acquire an overall Aharonov-Bohm phase

$$
\lim \operatorname{tr}\left\langle A_{a_{1}, a_{2}, \cdots, a_{n}}^{\nu}\left(\Sigma_{1}^{*}, \Sigma_{2}^{*}, \cdots, \Sigma_{n}^{*}, x_{0},\left\{l_{P}\right\} ; C\right)\right\rangle=\left(\frac{1}{n_{\nu}}\right) \chi^{\nu}\left(a_{1} a_{2} \cdots a_{n}\right) .
$$

With our implicit choice of $\left\{l_{P}\right\}$ in FIG. 5, one finds, contrary to the claim made in Ref. [15], that (in three spacetime dimensions) there is a higher order correction term:

$$
\begin{aligned}
& \lim \operatorname{tr}\left\langle A_{a_{1}, a_{2}, \cdots, a_{n}}^{\nu}\left(\Sigma_{1}^{*}, \Sigma_{2}^{*}, \cdots, \Sigma_{n}^{*}, x_{0},\left\{l_{P}\right\} ; C\right)\right\rangle \\
= & \left(\frac{1}{n_{\nu}}\right) \chi^{\nu}\left(a_{1} a_{2} \cdots a_{n}\right)+\sum_{g} O\left(\exp \left(8 \beta\left(\operatorname{Re} \chi^{(R)}(g)-n_{R}\right)\right)\right) \chi^{\nu}\left(g a_{1} g^{-1} a_{2} \cdots a_{n}\right),
\end{aligned}
$$

where $n_{\nu}\left(n_{R}\right)$ is the dimension of the representation $\nu(R)$. The second term on the r.h.s. (the higher order correction term) shows that coherence of the strings has been spoiled. 
(Cf. Eq. (20).) Taken at face value, our results seem to suggest that, because of quantum fluctuations, construction of order parameters for non-Abelian gauge theories is a hopeless enterprise. Of course, this conclusion is only an artifice of the particular choice of $\left\{l_{P}\right\}$. Let us look at the problem more closely. Since conjugation of some (but not all) of the plaquettes of a string requires the dynamical propagation of strings carrying the commutator of the various inserted fluxes, it is reasonable to believe that configurations of this type are energetically costly. For this reason, only conjugation of the calibrated flux of a whole string loop deserves attention. Here comes the question. With an ingenious choice of $\left\{l_{P}\right\}$, can one prevent vacuum fluctuations from conjugating the inserted flux of a whole string loop at a low energetic cost? The answer is no. Since all the tails, $\left\{l_{P}\right\}$, originate from the base point, quantum fluctuations can always conjugate the flux of a whole string loop just by flipping all the links from which the tails leave the base point. This corresponds to the picture in which a virtual string loop wraps around all the calibrating and measuring particles. Fortunately, this only leads to a redefinition of the basis that we use for both calibration and measurement and does not affect the result of our experiment.

The relevant question really is: Are there choices of $\left\{l_{P}\right\}$ by which one can prevent energetically inexpensive vacuum fluctuations from conjugating the inserted flux of a whole string loop without affecting the Wilson loop? The answer is yes. As emphasized in Section 3, any experimental determination of the non-Abelian Aharonov-Bohm factor essentially proceeds in two stages: calibration and measurement. Unless the two are done in a coordinated manner, it is entirely understandable that one may be fooled by quantum fluctuations. The idea is that quantum fluctuations in FIG. 4 conjugate the calibrated flux without affecting the measuring apparatus, thus preventing the recovery of the calibrated flux in the measurement. One can also consider an analogous process in which quantum fluctuations affect the measuring apparatus but not the calibrating apparatus. This would correspond to the configuration depicted in FIG. 6 where the worldline of the vortex-antivortex pair winds around the Wilson loop. Note that the configuration in FIG. 6 is, in fact, a smooth deformation of that of FIG. 4. In both figures, the vortex-antivortex worldline has a non-trivial 
linking number with the union of the Wilson loop,$\left\{l_{P}\right\}$. and the string loop.

Recall that in the original choice of $\left\{l_{P}\right\}$ by Alford et al., all the tails run along a chain of links from $x_{0}$ to $y_{0}$ which is not on the Wilson loop. Physically, this essentially means that the particles used for calibration and the subsequent measurement are kept in separate boxes. (We can regard a portion of the Wilson loop as the box for storing particles for subsequent measurement.) The problem is: a virtual string loop may nucleate, wrap around one of the boxes and re-annihilate. If this happens, the state of charged particles contained in the box wrapped around by the string loop will change relative to those in the other box. Since we are using particles in a particular box for calibration and those in the other box for subsequent measurement. Clearly, we get different answer for the two experiments. This is why this choice of $\left\{l_{P}\right\}$ does not work.

\section{The Resolution}

Having observed this point, the resolution is simple. We shall first present our resolution from a mathematical point of view and then back it up with physical intuition. First, note that if the Wilson loop and the path of calibration were the same, i.e. $C=l_{P} P l_{P}^{-1}$ for some $P$, the Wilson loop would trivially recover the calibrated element. A moment of thought reveals that the key problems are: on the one hand, all the tails must merge to the base point; on the other hand, since we take the limit that the closest approach of $C$ to $\Sigma^{*}$ approaches infinity in the definition of the order parameter (i.e., long range experiments), the tails $l_{P}$ 's inevitably contain links that are not on the Wilson loop. Quantum mechanical fluctuations of those links affect the calibration apparatus but not the measurment apparatus. The original construction is particularly vulnerable because there is a single link that belongs to all tails but is not on the Wilson loop. Branching may help reduce its vulnerability. Moreover, it may be a good idea for at least some of the tails to be initially on our Wilson loop, even though they must eventually branch out from it. Consider the configuration shown in FIG. 7 where the tails are chosen in such a way that many of them beginning from the base point 
are on our Wilson loop initially and branch out one by one from it. In what follows, we argue that this construction overcomes all difficulties caused by quantum fluctuations. In order for quantum fluctuations to affect the calibration but not the measurement, links on the Wilson loop must not be excited. Since by construction the tails that branch out from the Wilson loop never intersect one another after their branching out, to achieve overall conjugation of the flux of string loops, we must then flip a link in each tail after it has branched out. Since the number of tails that branch out goes to infinity as $\Sigma^{*}$ and $C$ get large, we must excite a large number of links. (See FIG. 7.) Such configurations have large actions and their contribution to the partition function is suppressed by factors proportional to the system size (e.g., the length of the Wilson loop). Our conclusion is that there is no energetically inexpensive way of conjugating the flux of a whole string loop without affecting the Wilson loop.

The above paragraph requires a severe qualification. It is important for our choice of the Wilson loop not to come close to retracing itself. Otherwise, it is possible for quantum fluctuations to affect the measuring but not the calibrating apparatus. Suppose the Wilson loop is chosen to be of the shape of a tennis racket. i.e. it runs along a long chain $L$ of links from the base point $x_{0}$ to a point $y_{0}$ and goes around various vortices of interest before coming back to $y_{0}$ where it retraces $L$ back to $x_{0}$. The tails are chosen such that many of them initially follow the chain $L$ of links on the Wilson loop, but finally branch out from $L$ one by one. Now the Wilson loop is vulnerable to quantum fluctuations: A worldline of virtual vortex can be linked to the Wilson loop near to $y_{0}$. With the choice of the Wilson loop in FIG. 8, the worldline of the virtual vortex, and therefore the energetic cost one pays in conjugating the measured flux relative to its calibrated value, is small.

There are strong physical motivations for our new choice of $\left\{l_{P}\right\}$. That the tails initially follow the Wilson loop corresponds to the physical picture that we keep the charged particles that we use for both calibration and subsequent measurement in the same box. The portion of the Wilson loop that the tails initally lie on corresponds to the worldline of this box. The fact that we are calibrating every plaquette through which the string passes (stage one) 
means that we calibrate the string loop continuously over time from the base point. (We need to specify the whole worldsheet.) Since the particles for both calibration and subsequent measurement are stored in the same box, lots of tails branch out from the Wilson loop.

Moreover, it is crucial not to send out the two beams of particles for subsequent measurement (stage two) too closely spaced in time. Otherwise, (with the tennis racket choice in FIG. 8) quantum fluctuations will spoil our result: a virtual string loop may wrap around both beams. This will conjugate the measured flux. To avoid this phenomenon, a wave packet of some test particles (one of the two beams) should be kept in the box that store the particles (for both calibration and measurement) and interfered with another wave packet (the other beam) that traverses the string. This is the physics underlying our new choice of $\left\{l_{P}\right\}$.

Recall in the discussion in the last Section that it is always possible for a virtual string loop to wrap around just one of two beams that we use for subsequent measurement. (This is true even in the case of an Abelian gauge theory.) However, this incoherent effect will go away on average if we repeat independent identical experiments many times to extract the expectation value. (See Eq. (5) in Section 3 and Eq. (33) in Section 7.)

In conclusion, our construction coordinates the calibration and measurement processes, making it impossible for our results to be sabotaged by just a few small virtual string loops. As noted earlier, it is always possible for a virtual string loop to wrap around the box storing all the particles. This leads to no real change as calibration and measurement are affected in the same way. The calculation in the next subsection will verify our assertion that a careful coordination between calibration and measurement solves the problem of quantum fluctuations that has plagued all previous attempts to construct order parameters for nonAbelian gauge theories. 


\section{Calculation}

Having specified our choice of tails, we shall now prove that at weak coupling, Eq. (25) is unspoiled by higher order corrections. Let us first consider the vacuum expectation value of the untraced Wilson loop operator $\left\langle U^{(\nu)}\left(C, x_{0}\right)\right\rangle$. Totally analogous to the $Z_{2}$ gaugeHiggs theory, at weak couplings discussed in the Appendix, we can completely ignore the matter action without changing our conclusion. Besides, the link excitations are so heavily suppressed that our result will be dominated by configurations with a low density of excited links. Therefore, configurations where any two excited links share a plaquette can be safely ignored, thus enabling us to factorize the sum over the links, $U_{i j}$, into two parts: one over the links that are on $C$ and one over that are not. The contributions from the second part are cancelled by a corresponding sum in the denominator. Now the gauge action, being gauge invariant, can be expressed in terms of character functions and we obtain

$$
\left\langle U^{(\nu)}\left(C, x_{0}\right)\right\rangle=\frac{1}{Z} \sum_{V_{1}} \sum_{V_{2}} \sum_{V_{n}} D^{(\nu)}\left(V_{1} V_{2} \cdots V_{n}\right) \times \prod_{i=1}^{n} \sum_{\mu^{*}} C^{\mu^{*}}(\beta) \chi^{\mu^{*}}\left(V_{i}\right),
$$

where $Z$ is same as the numerator but without the factor $D^{(\nu)}$. As discussed before, when $F_{a}\left(\Sigma^{*}, x_{0},\left\{l_{P}\right\}\right)$ is inserted, the configuration at weak couplings that has none of its plaquette frustrated is an "a-forest" in which, roughly speaking, all the links that intersect the minimal surface with boundary $\Sigma^{*}$ are of flux $a$. (FIG. 3.) Suppose the Wilson loop links once with the string loop. Exactly one of its links, $l$ (say with a flux $V_{l}$ ), is an $a$-forest link. So, its parallel neighbors are all of flux $a$ and any plaquette containing $l$ and one of its parallel neighbor will be of flux $a^{-1} V_{l}$. We can take this into account simply by defining a new variable $V_{l}^{\prime}=a^{-1} V_{l}$. Thus, in Eq. (29) we replace $D^{(\nu)}\left(V_{1} V_{2} \cdots V_{n}\right)$ by $D^{(\nu)}\left(V_{1} V_{2} \cdots V_{l-1} a V_{l}^{\prime} \cdots V_{n}\right)$ and $V_{l}$ by $V_{l}^{\prime}$ elsewhere. It follows from the orthogonality relations between matrix elements of irreducible representations,

$$
\int d U D_{i j}^{(\nu)}(U) D_{k l}^{(\mu) *}(U)=\frac{1}{n_{\nu}} \delta^{\mu \nu} \delta_{i k} \delta_{j l}
$$

that Eq. (25) survives higher order corrections. 


\section{E. More on Virtual Vortex Loops}

It is important to note that the a-forest, which is bounded by an inserted string worldsheet, is unphysical and can be moved around. We leave it as an exercise for the reader to show that the effect of the virtual vortex loop shown in FIG. 9 can be safely ignored. Note that the virtual vortex loop has trivial linkage with the union of the Wilson loop, the tails and the inserted string.

Incidentally, a careful choice of $\left\{l_{P}\right\}$ and the Wilson loop $C$ taken together will also maintain the quantum coherence between various string loops. A bad choice of $C$ by which quantum coherence is spoiled is shown in FIG. 10. A virtual vortex worldline conjugates the measured flux of one vortex relative to another. As a comparison, in FIG. 11, we show a choice of $C$ which would preserve quantum coherence. Let us consider paths which begin and end at points on the Wilson loop. Some of those paths (e.g. $P_{p q}$ ) are contractible. Some (e.g. $P_{y z}$ ) are not. We can classify paths into classes in which they are smoothly

deformable into one another. Let us consider the topologically non-trivial classes of paths. If the minimal lengths of all such paths diverge as $\Sigma_{1}^{*}, \Sigma_{2}^{*}$ and $C$ get large, then coherence of the inserted flux between the two vortices can be maintained. This is because quantum fluctuations that would conjugate the flux of one string relative to another are energetically costly and can, therefore, be safely ignored. Consequently, for a charged particle traversing $n$ coherent strings in succession, we obtain

$$
\begin{aligned}
\left\langle A_{a_{1}, a_{2}, \cdots, a_{n}}^{\nu}\left(\Sigma_{1}^{*}, \Sigma_{2}^{*}, \cdots, \Sigma_{n}^{*}, x_{0},\left\{l_{P}\right\} ; C\right)\right\rangle & =\frac{\left\langle F_{a_{1}, a_{2}, \cdots, a_{n}}\left(\Sigma_{1}^{*}, \Sigma_{2}^{*}, \cdots, \Sigma_{n}^{*}, x_{0},\left\{l_{P}\right\}\right) U^{\nu}(C)\right\rangle}{\left\langle F_{a_{1}, a_{2}, \cdots, a_{n}}\left(\Sigma_{1}^{*}, \Sigma_{2}^{*}, \cdots, \Sigma_{n}^{*}, x_{0},\left\{l_{P}\right\}\right)\right\rangle\left\langle\operatorname{tr} U^{\nu}(C)\right\rangle} \\
& =\left(\frac{1}{n_{\nu}}\right) \chi^{\nu}\left(a_{1} a_{2} \cdots a_{n}\right) .
\end{aligned}
$$

\section{GAUGE FIXING}

We have considered a discrete non-Abelian gauge group $G$ which arises as a result of the spontaneous symmetry breakdown of a simply-connected group $K$. We have successfully 
proved that in a free $G$ charge phase, with a careful choice of $\left\{l_{P}\right\}$, Eq. (25) remains valid even when quantum fluctuations are fully taken into account. One might naively expect that the operator $A_{a}^{\nu}\left(\Sigma^{*}, x_{0},\left\{l_{P}\right\} ; C\right)$ in Eq. 25) is the order parameter for non-Abelian gauge theories.

However, there are still complications in the study of the symmetry breakdown: $G \rightarrow H$. For one thing, with the breaking of $G$ into $H$, an irreducible representation $\nu$ of $G$ is typically reducible in $H$. In general, only one of the irreducible representations of $H$ that is contained in $\nu$ will dominate the asymptotic behavior of $W(C)$ when $C$ is large. Since particles in different irreducible representations of $H$ in the decomposition can be resolved from one another, it makes no sense to normalize them by the same factor $\operatorname{tr} U^{\nu}\left(C, x_{0}\right)$ in the denominator of the first line of Eq. (25). The correct thing to do is to consider each irreducible representation of $H$ separately and normalize the matrix elements (for each irreducible representation) by dividing the trace over the particular representation that one is working with.

There is a more serious problem. Unless $H$ is in the center of $G$, it does not make any sense to talk about irreducible representations of $H$ without some gauge fixing scheme. In particular, when $G$ is spontaneously broken into a non-normal subgroup $H$, it is easy to see that our original set of order parameters does not work. A gauge transformation by $g \in G$ at $x_{0}$ in Eq. (25) shows that

$$
\left.A_{g h g^{-1}}^{\nu}\left(\Sigma^{*}, x_{0},\left\{l_{P}\right\} ; C\right)\right\rangle=U^{\nu}(g) A_{h}^{\nu}\left(\Sigma^{*}, x_{0},\left\{l_{P}\right\} ; C\right) U^{\nu}\left(g^{-1}\right)
$$

The order parameters $A^{\nu}$ for $h$ and $g h g^{-1}$ are not independent and there is no way for us to distinguish the behavior of a string $h \in H$ with one $g h g^{-1} \notin H$. The way out is to perform gauge fixing. (To be more precise, the embedding the unbroken group $H$ in $G$ changes as $\phi$ varies. One should take this into account and consider $H$ as a function of $\phi$, i.e., $H(\phi)$.) Suppose $G$ acts transitively on the Higgs $\phi$. (We believe that the requirement that $G$ acts transitively on $\phi$ is only a working assumption. For configurations of finite energy, the Higgs field necessarily approaches its vacuum expectation value at spatial infinity and our gauge 
fixing procedure is well defined.) Without loss of generality, one can consider the gauge fixed insertion operator, $F_{a}\left(\Sigma^{*}, x_{0},\left\{l_{P}\right\}, \phi_{0}\right)$, where the Higgs field $\phi=\phi_{0}$. When this operator is inserted in a Green's function with gauge invariant operators, its gauge invariant part will be projected out. Thus, it will have the same effect as the gauge invariant operator, $\frac{1}{|H|} \sum_{h \in H(\phi)} F_{h a h^{-1}}\left(\Sigma^{*}, x_{0},\left\{l_{P}\right\}, \phi\right)$.

Even in the case that $H$ is normal in $G$ but not in the center of $G$, it is still necessary to gauge fix $\phi=\phi_{0}$ for the untraced Wilson loop operator $U^{\nu}(C)$. The reason is that, without gauge fixing, $U^{(\nu)}\left(C, x_{0}\right) \rightarrow D^{(\nu)}(g) U^{(\nu)}\left(C, x_{0}\right) D^{(\nu)}\left(g^{-1}\right)$ under a global gauge transformation by $g$. The expectation value $\left\langle U^{(\nu)}\left(C, x_{0}\right)\right\rangle$ is invariant under $G$. By the Schur's lemma, $\left\langle U^{(\nu)}\left(C, x_{0}\right)\right\rangle=\lambda I$ for some $\lambda$. The interpretation is clear: an irreducible representation of $G$ is typically reducible in $H$. In the absence of gauge fixing, it would not be possible for us to resolve the various irreducible representations of $H$ in the decomposition.

Therefore, we should always gauge fix $\phi=\phi_{0}$ and consider $U^{(\nu)}\left(C, x_{0}, \phi_{0}\right)$. From now on, we will only be interested in gauge transformation by the unbroken group $H\left(\phi_{0}\right)$. Let us decompose the representation $(\nu)$ into irreducible representations $\left(\mu_{1}\right),\left(\mu_{2}\right), \cdots,\left(\mu_{n}\right)$ of $H$ and the representation space $V=V_{1} \oplus V_{2} \cdots \oplus V_{n}$. The leading non-trivial contribution to $\left\langle U^{(\nu)}\left(C, x_{0}, \phi_{0}\right)\right\rangle$ must arise when one of the links on $C$ takes a value $h$ in the unbroken group $H$. (If it takes a value $g \notin H$, some matter fields are excited. Thus, its contribution to $\left\langle U^{(\nu)}\left(C, x_{0}, \phi_{0}\right)\right\rangle$ must be suppressed.) By gauge fixing $\phi=\phi_{0}$, we can disentangle various irreducible representations of $H$ from one another and see clearly that the symmetry group has been reduced to $H$. In other words, in the free $H$ charged phase and with gauge fixing, we obtain again Eq. (25) for a representation of $H$ (rather than $G$ ). As discussed in Section 4, a string with flux $a \notin H$ is now bounded to a domain wall which decays to a composite string with flux $a b^{-1} \in H$. Thus, the Aharonov-Bohm phases will give elements of $H$ only and this shows clearly that the unbroken group is $H$. From that point on, there is no obstacle to repeating our original analysis to study any symmetry breaking of $H$ at an even lower energy scale. 


\section{DISCUSSIONS}

\section{A. Gauge-invariant Formulation}

Note that the operators $F_{a}\left(\Sigma^{*}, x_{0},\left\{l_{P}\right\}, \phi_{0}\right)$ and $U^{\mu}\left(C, x_{0}, \phi_{0}\right)$ we use in the definition of our order parameters are not gauge invariant under $H$. However, it is possible for us to redefine the order parameters in terms of quantities which are invariant under $H$. This is done by tracing over an irreducible representation $(\mu)$ of $H$ in the Wilson loop operator and for the insertion of strings, we use the operator $\frac{1}{|H|} \sum_{h \in H\left(\phi_{0}\right)} F_{h a h^{-1}}\left(\Sigma^{*}, x_{0},\left\{l_{P}\right\}, \phi_{0}\right)$. In conclusion, we propose that

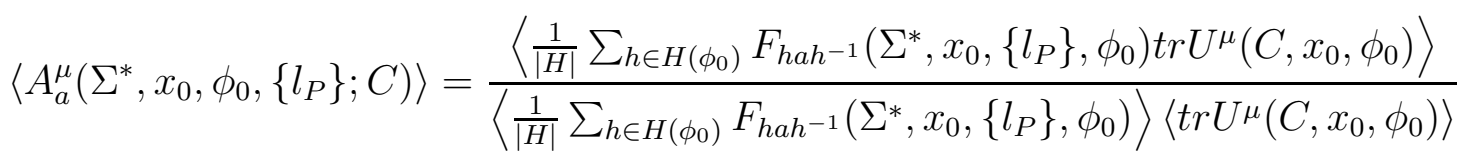

is an order parameter for non-Abelian gauge theories. At first sight, our order parameters may appear to be redundant because, their definition seems to require a knowledge of the identity of the Higgs field and the action of the gauge group on it. Doesn't it mean that

we already know what the unbroken group is? Our view point is that one should think of $\phi$ only as a candidate for the Higgs field rather than the Higgs field itself. We are testing with our order parameters if a Higgs phenomenon has occurred. The actual realization of the gauge symmetry depends on the values of the coupling constants in our theory.

\section{B. Example: $S_{3} \rightarrow Z_{2}$}

Since the above discussion is rather abstract, one desires an explicit model in which the bahavior of the order parameter $A_{a}^{\nu}(\Sigma, C)$ can be studied analytically. However, there are some technical difficulties. A matter action on a lattice that we find tractable is of the form

$$
S_{\mathrm{Higgs}}=-\sum_{\mu} \gamma_{\mu} \sum_{l}\left(\chi^{\mu}\left(\left(\phi^{-1} U \phi\right)_{l}\right)+c . c .\right)
$$

where the sum runs over all irreducible representations of $G$. Perturbative methods can be used to analyze this model when some of the $\gamma_{\mu}$ 's are large $(\gg 1)$ while all others are small 
$(\ll 1)$. In the weak coupling limit $\gamma_{\mu} \rightarrow \infty, \phi^{-1} U \phi$ becomes restricted to the kernel of the representation $(\mu)$ at each link and $U_{P}$ takes value in the kernel at each plaquette. In other words, $G$ is spontaneously broken into the subgroup $H=\operatorname{Ker}\left(D^{\mu}\right)$, which is normal in $G$. Indeed, the breaking of $G$ into any normal subgroup can be obtained by choosing some $\gamma_{\mu}$ 's to be large. Of course, it is still conceivable that at immediate couplings, a more general symmetry breaking pattern can occur and a non-normal subgroup may happen to be the unbroken group. However, at immediate couplings, our perturbative methods clearly break down and there is no simple way of analyzing the result other than numerical methods.

It would be very helpful if one can come up with a more general tractable lattice action in which a non-Abelian group is spontaneously broken into a non-normal subgroup. Let the matter field $\phi$ take values in the left coset of $H$ (denoted by $G / H$ ) and consider the action of the form

$$
S=-\beta \sum_{P}\left(\chi^{R}\left(U_{P}\right)+\text { c.c. }\right)-\gamma \sum_{l} F\left(\phi_{i}, U_{i j} \phi_{j}\right)
$$

where $F$ is a mapping from $G / H \times G / H$ to real numbers. $F$ has to respect gauge invariance. i.e. $F\left(g g_{1} H, g g_{2} H\right)=F\left(g_{1} H, g_{2} H\right)$. Besides, $F(H, H) \geq F\left(g_{1} H, g_{2} H\right)$ for all $g_{1}, g_{2} \in G$. Let us apply our formalism to the case of the symmetry breakdown of $S_{3}$ to $Z_{2}$. Without loss of generality, let $H=\{e,(12)\}$. Suppose $F(H, H)=1$ and $F(H,(23) H)=F(H,(13) H)=$ 0. We shall assume without proof that there exist convergent weak and strong coupling expansion schemes for the matter action of Eq. (35).

(1) $\beta \gg 1, \gamma \ll 1$

Just like the $Z_{2}$ gauge-Higgs system discussed in the Appendix, the matter action can be safely ignored. $\left\langle F_{a}\left(\Sigma^{*}\right)\right\rangle$ is dominated by an $a$-forest. Our order parameters show that the theory is in a free $S_{3}$ charge phase.

(2) $\beta, \gamma \gg 1$

Now the leading non-trivial contribution to $\langle W(C)\rangle$ arises when one of the links on $C$ has $U_{l}=(12)$. (A configuration with one of the links on $C$ taking a value $U_{l} \neq e$ or (12) excites the matter field and its contribution is thus severely suppressed.) It is easy to check 
that the two-dimensional irreducible representation of $S_{3}$ is decomposed into a trivial and a non-trivial representations of $Z_{2}$. With gauge fixing, the two representations can easily be resolved from each other. While $F_{(12)}$ inserts a stable string loop, other $F_{a}$ insert string loops which are bounded by domain walls. Thus, the theory is in a free $Z_{2}$ charge phase.

From the non-analytic behavior of the order parameters across the phase boundary, we see that there is a symmetry breaking from $S_{3}$ to $Z_{2}$.

\section{Vacuum Overlap Order Parameters}

Other order parameters for gauge theories have also been previously proposed. (For a review, see Ref. [25].) One promising probe of the phase structure of a gauge theory is the vacuum overlap order parameters (VOOP) proposed by Fredenhagen and Marcu [13. Suppose a matter field $\Phi^{(\mu)}$ which tranforms irreducibly under a gauge group $G$. Choose a path $P_{x, y}$ which connects two widely separated points $x$ and $y$ and consider the gaugeinvariant operator

$$
K^{(\mu)}\left(x, y, P_{x, y}\right)=\Phi_{x}^{(\mu) \dagger} D^{(\mu)}\left(\left(\prod_{l \in P_{x, y}} U_{l}\right)\right) \Phi_{y}^{(\mu)} .
$$

If the gauge group $H$ is unbroken, the field $\Phi^{\dagger}$ should create a stable particle which will propagate between $x$ and $y$. Therefore, we have

$$
\left\langle K^{(\mu)}\left(x, y, P_{x, y}\right)\right\rangle \sim \exp \left(-M_{\text {ren }}^{(\mu)} L(P)\right) \exp \left(-M_{\text {dyn }}^{(\mu)}|x-y|\right)
$$

where $M_{\text {ren }}^{(\mu)}$ is the renormalised mass of the classical source of charges propagating along $P$, $M_{\mathrm{dyn}}^{(\mu)}$ is the dynamical mass of the stable particle created by $\Phi^{(\mu) \dagger}, L(P)$ is the length of $P$ and $|x-y|$ is the distance from $x$ to $y$. If the representation $(\mu)$ is confined or screened, there will be no dynamical propagation of stable charged particles. Thus, we expect that $\left\langle K^{(\mu)}\left(x, y, P_{x, y}\right)\right\rangle$ is independent of $|x-y|$ for large separation. i.e. $M_{\mathrm{dyn}}^{(\mu)}=0$. Fredenhagen

and Marcu proposed that $M_{\mathrm{dyn}}^{(\mu)}>0$ is the criterion for $\Phi^{\dagger}$ to create a free charge. Their construction is highly similar to our order parameters. When the gauge group is broken into 
a subgroup $H$, the complications in disentangling various irreducible representations of $H$ in the decomposition of an irreducible representation of $G$ discussed in the last section also arise here. We expect the resolution is again gauge fixing. It would be interesting to work it out. Finally, we remark that application of the VOOP to the study of partial symmetry breakdown has been considered in the explicit example of the Georgi-Glashow model [26].

\section{Phase Transition Without Symmetry breaking?}

It was suggested in Ref. [15] that there is a possibility of having a phase transition without a change in the symmetry group. Recall that generally just one irreducible representation of $H$ will dominate the asymptotic behavior of $U^{\nu}$. Alford et al. proposed that in some parameter space of the theory a "crossover" may occur, where this representation changes. We are not sure whether such an interesting phenomenon is possible.

\section{E. Conclusions}

Our order parameters are useful for studying the symmetry breakdown of non-Abelian gauge theories. While we have concentrated our discussion on discrete group theories, we emphasize that the idea of the Aharonov-Bohm order parameter is rather general. The assumptions of the existence of topologically stable flux tubes and the symmetry group being discrete may be relaxed. The subtleties of quantum fluctuations and gauge fixing are intrinsic properties of non-Abelian gauge theories.

We emphasize that, after gauge fixing, vortices of conjugate but different fluxes should be regarded as non-identical. The coherence of the fluxes between various strings is characteristic of non-Abelian gauge theories. Quantum fluctuations tend to destroy these two important features. In our construction of the order parameter, we show how these problems can be overcome by a careful coordination between the calibrating and measuring processes.

When a gauge group $G$ is broken into $H$, an irreducible representation of $G$ is typically reducible in $H$. Particles in different irreducible representations of the decomposition can 
be resolved from one another. In order to isolate the behavior of a particular irreducible representation of $H$, it is crucial to gauge fix the Higgs field $\phi=\phi_{0}$. This simple but crucial point has also been largely overlooked in previous works. We also sketch briefly the application of the Aharonov-Bohm order parameters to study the symmetry breaking of $S_{3}$ to $Z_{2}$ and discuss the vacuum overlap order parameters suggested in the literature.

We would also like to remark that a Chern-Simons term can be added to the action of a gauge theory in $2+1$ dimensions. Our construction remains to be generalized to this case. It is also of interest to note that linked Wilson loops are useful as order parameters for a Maxwell-Chern-Simons-Higgs system. In the unbroken Chern-Simons phase, matter charges are attached with fluxes, thus experiencing Aharonov-Bohm interactions with one another. There is, however, no such Aharonov-Bohm interactions in the Chern-Simons Higgs phase because the Higgs mechanism removes the fluxes that are attached to the matter charges in the unbroken phase [27]. Finally, from a mathematical point of view, it is conceivable that these types of non-local objects involved in the construction of order parameters may give rise to interesting topological invariants [28].

\section{ACKNOWLEDGMENTS}

We are indebted to J. Preskill for bringing the problem of quantum fluctuations in the context of order parameters for non-Abelian gauge theories to our attention and for stimulating discussions at various stages of this work. Numerous helpful conversations with S. Adler, M. Bucher, H. F. Chau, K.-M. Lee, J. March-Russell, P. McGraw, F. Wilczek, M.

de Wild Propitius and P. Yi are also gratefully acknowledged. This work was supported in part by DOE DE-FG02-90ER40542.

\section{APPENDIX: EXAMPLE: $Z_{2}$ GAUGE-HIGGS SYSTEM}

Here we review the application of the order parameter to a simple model in the work of Preskill and Krauss [6]: $Z_{2}$ lattice gauge theory coupled to a $Z_{2}$ spin system. The degrees 
of freedom of the model are gauge variables

$$
U_{l} \in Z_{2} \equiv\{1,-1\}
$$

residing on links (labeled by $l$ ) of a cubic four-dimensional spacetime lattice and spin variables

$$
\phi_{i} \in Z_{2} \equiv\{1,-1\}
$$

residing on sites (labeled by $i$ ). The partition function of the theory is

$$
Z=\sum_{\{U\}\{\phi\}} e^{-S}
$$

where the Euclidean action is

$$
S=S_{\text {gauge }}+S_{\text {spin }}
$$

where

$$
S_{\text {gauge }}=-\beta \sum_{P} U_{P}
$$

and

$$
S_{\text {spin }}=-\gamma \sum_{l}(\phi U \phi)_{l}
$$

where $U_{P}=\prod_{l \in P} U_{l}$ associates with each elementary plaquette $P$ the product of the four gauge variables $U_{l}$ 's sitting on its links, and $(\phi U \phi)_{i j}=\phi_{i} U_{i j} \phi_{j}$, for each pair of neighboring sites. The action is invariant under the $Z_{2}$ gauge transformation defined by

$$
\eta_{i} \in Z_{2} \equiv\{1,-1\}
$$

where the variables transform as

$$
\phi_{i} \rightarrow \eta_{i} \phi_{i}, U_{i j} \rightarrow \eta_{i} U_{i j} \eta_{j}
$$

Note that the gauge variable $U_{l}$ is invariant under a non-trivial global gauge transformation $\eta_{i}=-1$, but the spin variable $\phi_{i}$ is not. The spin variable is, therefore, a matter field 
with a non-trivial $Z_{2}$ charge and we would like to determine if there is a $Z_{2}$ superselection rule.

Now we must consider how the operator $F\left(\Sigma^{*}\right)$ is to be defined on a lattice. Recall that inserting $F\left(\Sigma^{*}\right)$ into a Green's function is supposed to be equivalent to introducing a classical cosmic string source on the world sheet $\Sigma^{*}$. In a $(3+1)$-dimensional lattice, we consider $\Sigma^{*}$ to be a closed surface made up of plaquettes of the dual lattice. There is a set $\Sigma$ of plaquettes of the original lattice that are dual to the plaquettes of $\Sigma^{*}$. (It is easier to visualize in three spacetime dimensions. Then $\Sigma^{*}$ is a closed path made up of links in the dual lattice. Each link of $\Sigma^{*}$ is dual to a plaquette of the original lattice. See FIG. 3.) The operator $F\left(\Sigma^{*}\right)$ modifies the gauge action of these plaquettes:

$$
-\beta U_{P} \rightarrow \beta U_{P}, P \in \Sigma
$$

This is equivalent to flipping the sign of $\beta$ in these plaquettes.

Let us consider a pure gauge theory first. $\Sigma^{*}$ is the boundary of a set of cubes of the dual lattice. The insertion of $F\left(\Sigma^{*}\right)$ is equivalent to performing a singular gauge transformation

$$
U_{l} \rightarrow-U_{l}
$$

on all the links that are dual to those cubes. (As shown in FIG. 3, in three spacetime dimensions, $\Sigma^{*}$ is the boundary of a set of plaquettes in the dual lattice. The insertion of $F\left(\Sigma^{*}\right)$ is equivalent to performing singular gauge transformations $U_{l} \rightarrow-U_{l}$ on all the links dual to these plaquettes. Those links are marked by arrows.) So, $F\left(\Sigma^{*}\right)$ is just a change of variable and

$$
\left\langle F\left(\Sigma^{*}\right)\right\rangle=1
$$

The Wilson loop on the lattice is defined as

$$
W(C)=\prod_{l \in C} U_{l}
$$

where $C$ is a closed loop of links. If the surface $\Sigma^{*}$ and the loop $C$ have a linking number 1 , $F\left(\Sigma^{*}\right)$ flips the sign of one $U_{l}$ on $C$ and we find 


$$
\left\langle F\left(\Sigma^{*}\right) W(C)\right\rangle=-\langle W(C)\rangle .
$$

This show that $Z_{2}$ charge is not screened in a pure gauge system and a $Z_{2}$ cosmic string can be detected at long range by a $Z_{2}$ charge.

Let us now turn to the full theory: $Z_{2}$ gauge-Higgs system. This model is tractable because it can be analyzed by means of convergent perturbation expansions. The phase structure of this theory shown in FIG. 12 has previously been conjectured and confirmed by Monte Carlo simulations. Preskill and Krauss have shown that the order parameter $A(\Sigma, C)$ is an appropriate order parameter. To avoid overburdening the reader with technical details, we shall only present explicit calculations in two regions. These calculations are sufficient to prove their case.

(1) $\beta \gg 1, \gamma \ll 1$

In this region, $\exp (-\beta)$ and $\gamma$ are small. So, the gauge variables are hard to excite but the spin variables are easy. Therefore, configurations with only a small number of frustrated plaquettes will be important and we can expand the gauge action in powers of $\exp (-\beta)$. In effect, we are expanding in terms of world sheets of virtual strings. The matter action can be expanded, in powers of $\tanh \gamma$, as

$$
e^{-S_{\mathrm{spin}}}=N(\gamma) \prod_{l}\left[1+(\phi U \phi)_{l} \tanh \gamma\right] .
$$

Consider the leading non-trivial contribution to $\langle W(C)\rangle$. It is zeroth order in $\tanh \gamma$ and indifferent to spin frustrations. Therefore, we can safely ignore the matter action without changing our conclusion. Considering the gauge action alone, the leading non-trivial contribution to $\langle W(C)\rangle$ arises when one of the links on $C$ has $U_{l}=-1$. In four spacetime dimensions, this will frustrate six plaquettes that contain the link. (For ease of visualisation, the corresponding picture in three dimensions in which four plaquettes are frustrated is drawn in FIG. 13. It corresponds simply to the physical picture of having a small virtual vortex worldline linked to the Wilson loop.) Thus, we find

$$
\langle W(C)\rangle=\frac{\exp \left(-L\left(e^{-2 \beta}\right)^{6}+\cdots\right)}{\exp \left(+L\left(e^{-2 \beta}\right)^{6}+\cdots\right)}=\exp \left[-2 L\left(e^{-2 \beta}\right)^{6}+\cdots\right],
$$


where $L$ is the number of links on $C$. The exponentiation results from summing over the $L^{N} / N$ ! ways of flipping the sign of $N$ of the links on $C$. For $\left\langle F\left(\Sigma^{*}\right)\right\rangle$, the leading contribution is obtained by flipping all the links dual to the volume enclosed by $\Sigma^{*}$. Then $U_{P}=-1$ on the plaquettes dual to $\Sigma^{*}$ and $U_{P}=1$ elsewhere, so that no plaquette variables are frustrated. Let us call this set of links with flux -1 a "-1-forest". (See FIG. 3.) This -1-forest configuration, in which the gauge variables $U_{l}$ are flipped in a volume bounded by $\Sigma^{*}$, dominates $\left\langle F\left(\Sigma^{*}\right)\right\rangle$ because the gauge variables are ordered and costly to excite, while the spin variables are disordered and nearly indifferent to a flip in their nearest-neighbor couplings inside $\Sigma^{*}$. We expand the spin partition function with the plaquette variables frozen at these values to find

$$
\begin{aligned}
& \left\langle F\left(\Sigma^{*}\right)\right\rangle=\frac{\left[1-(\tanh \gamma)^{4}\right]^{A}}{\left[1+(\tanh \gamma)^{4}\right]^{A}} \\
& =\exp \left[-2 A(\tanh \gamma)^{4}+\cdots\right]
\end{aligned}
$$

Here summing over the spin variables around each plaquette on $\Sigma$ gives a factor $\frac{\left[1-(\tanh \gamma)^{4}\right]}{\left[1+(\tanh \gamma)^{4}\right]}$ and $A$ is the area of $\Sigma^{*}$. With the contribution to $A\left(\Sigma^{*}, C\right)$ being dominated by the -1 forest, a cosmic string has hair and we find

$$
\lim A\left(\Sigma^{*}, C\right)=-1
$$

if $\Sigma$ and $C$ have an odd linking number. Therefore, there is a $Z_{2}$ superselection rule. In other words, $Z_{2}$ is the manifest low energy symmetry group.

(2) $\beta, \gamma \gg 1$ Once again the leading non-trivial contribution to $\langle W(C)\rangle$ arises when one of the links on $C$ has $U_{l}=-1$. The only difference is that flipping $U_{l}$ now frustrates the spins on the link as well as the six plaquettes that contain the link. Therefore,

$$
\langle W(C)\rangle=\exp \left[-2 L\left(e^{-2 \beta}\right)^{6} e^{-2 \gamma}+\cdots\right]
$$

where $L$ is the length of $C$. The crucial difference from region (1) lies in the behavior of $\left\langle F\left(\Sigma^{*}\right)\right\rangle$. Since spin frustration is now costly, the leading contribution to $\left\langle F\left(\Sigma^{*}\right)\right\rangle$ no longer arises from a -1 -forest. A -1 -forest will frustrate spins in a volume bounded by $\Sigma^{*}$. The 
preferable configuration is to frustrate all plaquettes dual to $\Sigma^{*}$. This gives an area law decay

$$
\left\langle F\left(\Sigma^{*}\right)\right\rangle=\left(e^{-2 \beta}\right)^{A}+\cdots,
$$

where $A$ is the area of $\Sigma^{*}$.

Since the gauge variables $U_{l}$ 's deep inside the volume bounded by $\Sigma^{*}$ are unaffected by the insertion of $F\left(\Sigma^{*}\right)$, we see clearly that

$$
\lim \left\langle A\left(\Sigma^{*}, C\right)\right\rangle=1
$$

The interpretation is simple. Spontaneous symmetry breaking of $Z_{2}$ has occurred. Condensation of the matter field causes the string to become the boundary of a domain wall, but the wall is unstable and decays by nucleation of a loop of string. The inserted $Z_{2}$ string thus becomes bounded to another $Z_{2}$ string and the composite object then gives a trivial Aharonov-Bohm factor.

From the different behaviors in the two regimes, one concludes that $\left\langle A\left(\Sigma^{*}, C\right)\right\rangle$ is an appropriate order parameter for the $Z_{2}$ model. This result can be readily generalized to a lattice gauge theory with an arbitrary Abelian gauge group. 


\section{REFERENCES}

[1] G. 't Hooft, Nucl. Phys. B138, 1 (1978); B153, 141 (1979).

[2] A. Vilenkin, Phys. Reports 121, 263 (1985).

[3] J. Preskill, "Vortices and Monopoles," in Architecture of the Fundamental Interactions at Short Distances, ed. P. Romand and R. Stora (North-Holland, Amsterdam, 1987).

[4] Y. Aharonov and D. Bohm, Phys. Rev. 115, 485 (1959).

[5] L. M. Krauss and F. Wilczek, Phys. Rev. Lett. 62, 1221 (1989).

[6] J. Preskill and L. M. Krauss, Nucl. Phys. B341, 50 (1990).

[7] S. Coleman, J. Preskill and F. Wilczek, Nucl. Phys. B378, 175 (1992).

[8] M. Bucher, H.-K. Lo and J. Preskill, Nucl. Phys. B386, 3 (1992).

[9] M. Alford et al. , Phys. Rev. Lett. 64, 1632 (1990); 65, 668 (E); Nucl. Phys. B349, 414 (1991).

[10] M. Bucher, K.-M. Lee and J. Preskill, Nucl. Phys. B386, 27 (1992).

[11] F. A. Bais, P. van Driel and M. de Wild Propitius, Phys. Lett. B280, 63 (1992); Nucl. Phys. B393, 547 (1993); F. A. Bais and M. de Wild Propitius, Theor. Math. Phys. 98, 357 (1994).

[12] H.-K. Lo and J. Preskill, Phys. Rev. D48, 4821 (1993).

[13] K. Fredenhagen and M. Marcu, Comm. Math. Phys. 92, 81 (1983); Phys. Rev. Lett. 56, 223 (1986).

[14] M. Alford and J. March-Russell, Nucl. Phys. b369, 276 (1992).

[15] M. Alford, K.-M. Lee, J. March-Russell and J. Preskill, Nucl. Phys. B384, 251 (1992).

[16] H.-K. Lo, "Elusive Order Parameters for Non-Abelian Gauge Theories." IASSNS-HEP- 
$95 / 4$.

[17] P. A. M. Dirac, Proc. Royal. Soc. A133, 60 (1931).

[18] S. Elitzur, Phys. Rev. D12, 3978 (1975).

[19] M. Alford, S. Coleman and J. March-Russell, Nucl. Phys. B351, 735 (1991).

[20] F. A. Bais, A. Morozov and M. de Wild Propitius, Phys. Rev. Lett. 71, 2383 (1993).

[21] V. Poénaru and G. Toulouse, J. Phys. (Paris) 38, 887 (1977); F. Bais, Nucl. Phys. B170, 32 (1980).

[22] M. Bucher, Nucl. Phys. B350, 163 (1991).

[23] F. Wilczek and Y.-S. Wu, Phys. Rev. Lett. 65, 13 (1990).

[24] A related discussion was made in the private communications between M. Bucher and J. Preskill, Nov. 1992.

[25] K. Fredenhagen, "Particle Structure of Gauge Theories," in Fundamental Problems of Gauge Field Theory. ed. G. Velo and A. S. Wightman (Plenum, New York, 1986).

[26] T. Filk, K. Fredenhagen, M. Marcu, and K. Szlachányi, Phys. Lett. B217, 505 (1989).

[27] M. de Wild Propitius, Ph. D. thesis (unpublished).

[28] E. Witten, Comm. Math. Phys. 121, 331 (1989); A. S. Cattaneo, P. Cotta-Ramusino, A. Gamba and M. Martellini, Phys. Lett. B355, 245 (1995); D. Anselmi, hep-th/9504049 (unpublished). 


\section{FIGURES}

FIG. 1. (a) The test particles that we use for calibration and measurement are stored in separated boxes. This arrangement is vulnerable to quantum fluctuations. More concretely, a virtual string loop may nucleate, wrap around one of the boxes and annihilate, thus changing the state of the particles in one box but not the other. When we use test particles in the two boxes to determine the flux of a string loop, they give two different values (which are related by conjugation by the group element associated with the virtual string loop). (b) If the test particles for both calibration and subsequent measurement are stored in the same box, the problem disappears as any virtual string loop which affects the particles for calibration is going to affect those for subsequent measurement in the same way.

FIG. 2. Two consecutive Aharonov-Bohm experiments are performed to measure the flux of a vortex. The dashed line (with arrow) represents the worldline of a virtual vortex, which is linked to the union of the two Wilson loops and the vortex worldline of interest. The second measurement of the flux gives a value which is a conjugate of that of the first. i.e. quantum fluctuations render the flux of a string uncertain up to conjugation.

FIG. 3. The dashed line is $\Sigma^{*}$, comprised of links of the dual lattice. The plaquettes shown belong to $\Sigma$ and are dual to the links of $\Sigma^{*}$. The links marked by arrows are a-forest links. i.e. In the leading weak coupling expansion, they are of flux $a$.

FIG. 4. Suppose all $l_{P}$ merge together at some point $y_{0}$ not on the Wilson loop before reaching the base point. A worldline of virtual vortex conjugates the calibrated flux.

FIG. 5. Suppose the $l_{P}$ from each vortex merge together before reaching the basepoint. There exists a short worldline of virtual vortex (dashed line) which is topologically linked to the rest of the figure. Owing to quantum fluctuations, the calibrated flux of vortex 2 relative to that of vortex 1 is rendered uncertain up to conjugation. This would destroy the coherence of flux between the two vortices. 
FIG. 6. A deformation of the configuration shown in FIG. 6. The worldline of the virtual vortex now winds around the Wilson loop, thus affecting the measurement, but not the calibration apparatus.

FIG. 7. Lots of long tails initially lie on the Wilson loop $C$. They eventually branch out from it one by one and never intersect one another afterwards. Moreover, the Wilson loop never comes close to retracing itself. To conjugate the calibrated flux without affecting the measurement, each tail $l_{P_{i}}$ must be wrapped around by a virtual string loop after its branching out from the Wilson loop. Since the number of tails becomes large as $C$ and $\Sigma^{*}$ get large, such configurations are energetically costly. We, therefore, conclude that, with a coordinated choice of the Wilson loop and $\left\{l_{P}\right\}$, any energetically inexpensive excitation that affects the calibration process necessarily affects the measurement process and vice versa.

FIG. 8. The Wilson loop, $C$, is of the shape of a tennis racket with a long chain running from $x_{0}$ to $y_{0}$. Starting from the base point, lots of long tails initially lie on the long chain. They ultimately branch out from it one by one and never intersert one another thereafter. A virtual vortex worldline (the dashed line) winds around the Wilson loop, thus affecting the measurement process but not the calibration.

FIG. 9. The effect of the virtual vortex worldline (the dashed line) shown in the figure can be safely ignored. The "a-forest" is unphysical and can be moved around by gauge transformations. The virtual vortex worldline is unlinked to the rest of the figure.

FIG. 10. The Wilson loop winds around vortex 2 first and vortex 1 second. Suppose it is chosen such that it returns to the base point after winding around vortex 2. A virtual vortex worldline of small size can conjugate the measured flux of an inserted string relative to another.

FIG. 11. The path $P_{p q}$ joining the points $p$ and $q$ on the Wilson loop is contractible while there is topological obstruction to the shrinkage of the path $P_{y z}$ to a single point. 
FIG. 12. Phase diagram of the $Z_{2}$ gauge-Higgs system.

FIG. 13. The leading non-trivial contribution to $\langle W(C)\rangle$ has a single link flipped $\left(U_{l}=-1\right)$. Note that the Wilson loop and the virtual vortex world line (denoted by the dashed line with arrow) have a linking number one. 
This figure "fig1-1.png" is available in "png" format from: http://arxiv.org/ps/hep-th/9502080v2 


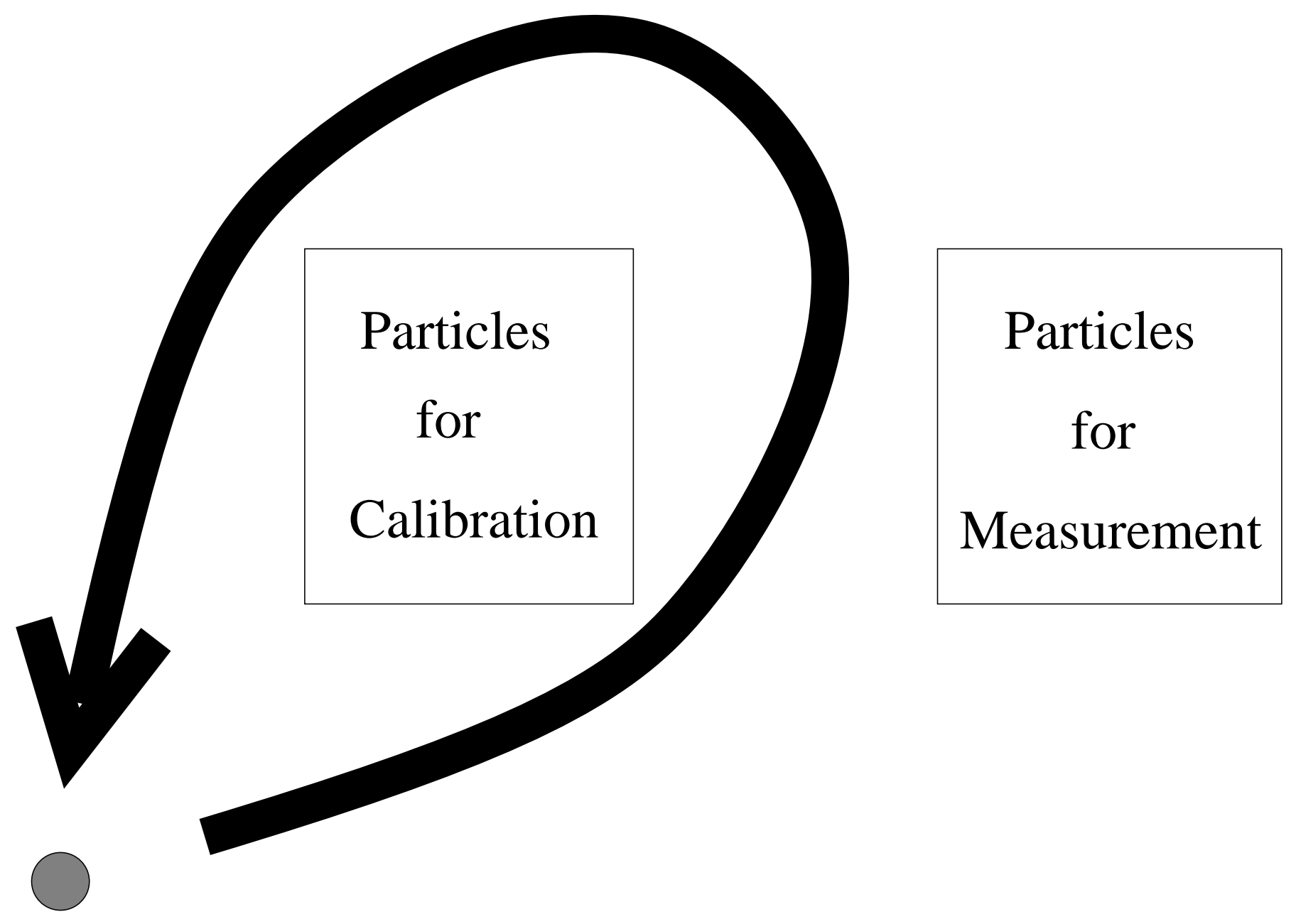




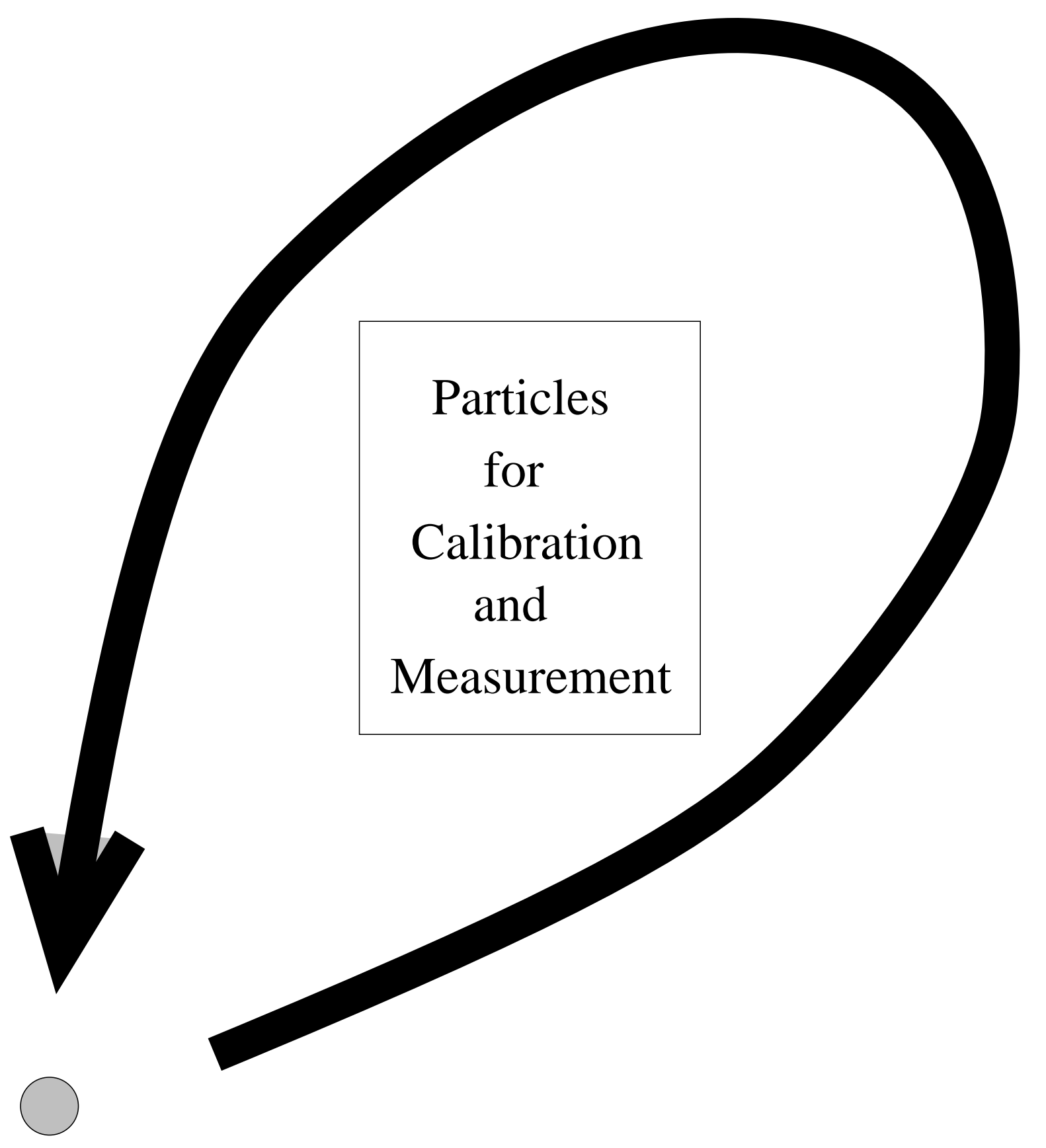


This figure "fig2-1.png" is available in "png" format from: http://arxiv.org/ps/hep-th/9502080v2 
This figure "fig1-2.png" is available in "png" format from: http://arxiv.org/ps/hep-th/9502080v2 
This figure "fig2-2.png" is available in "png" format from: http://arxiv.org/ps/hep-th/9502080v2 


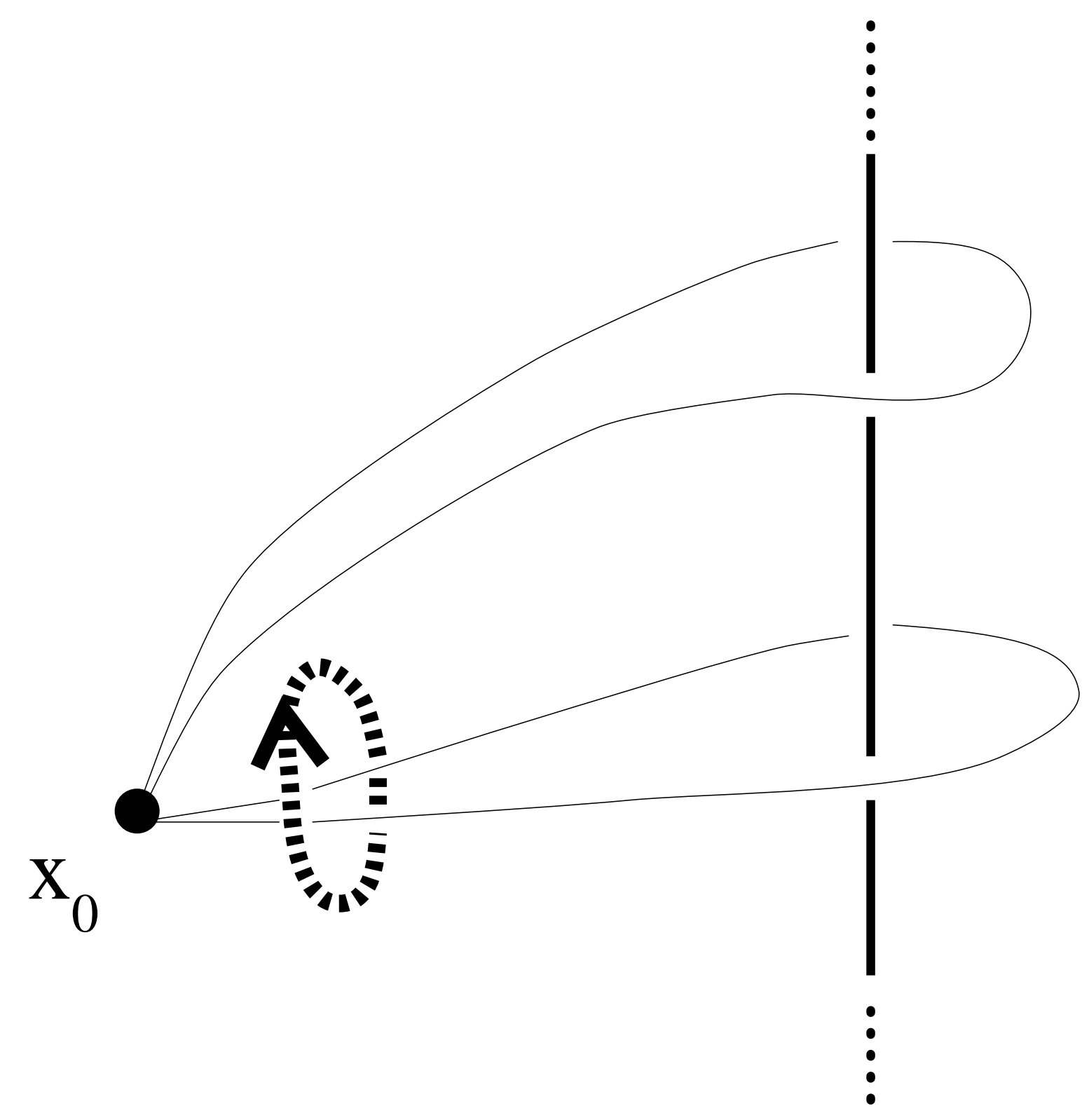


This figure "fig1-3.png" is available in "png" format from: http://arxiv.org/ps/hep-th/9502080v2 
This figure "fig2-3.png" is available in "png" format from: http://arxiv.org/ps/hep-th/9502080v2 


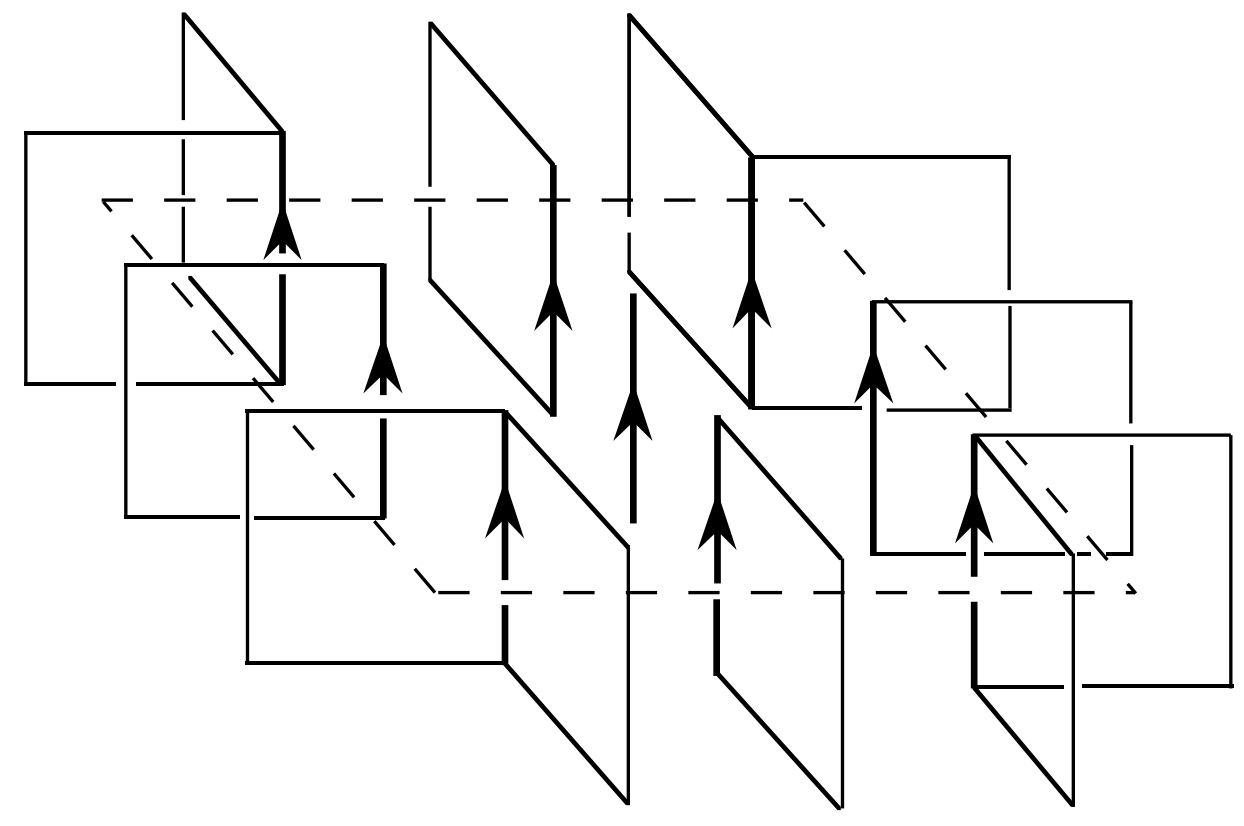



This figure "fig2-4.png" is available in "png" format from: http://arxiv.org/ps/hep-th/9502080v2 


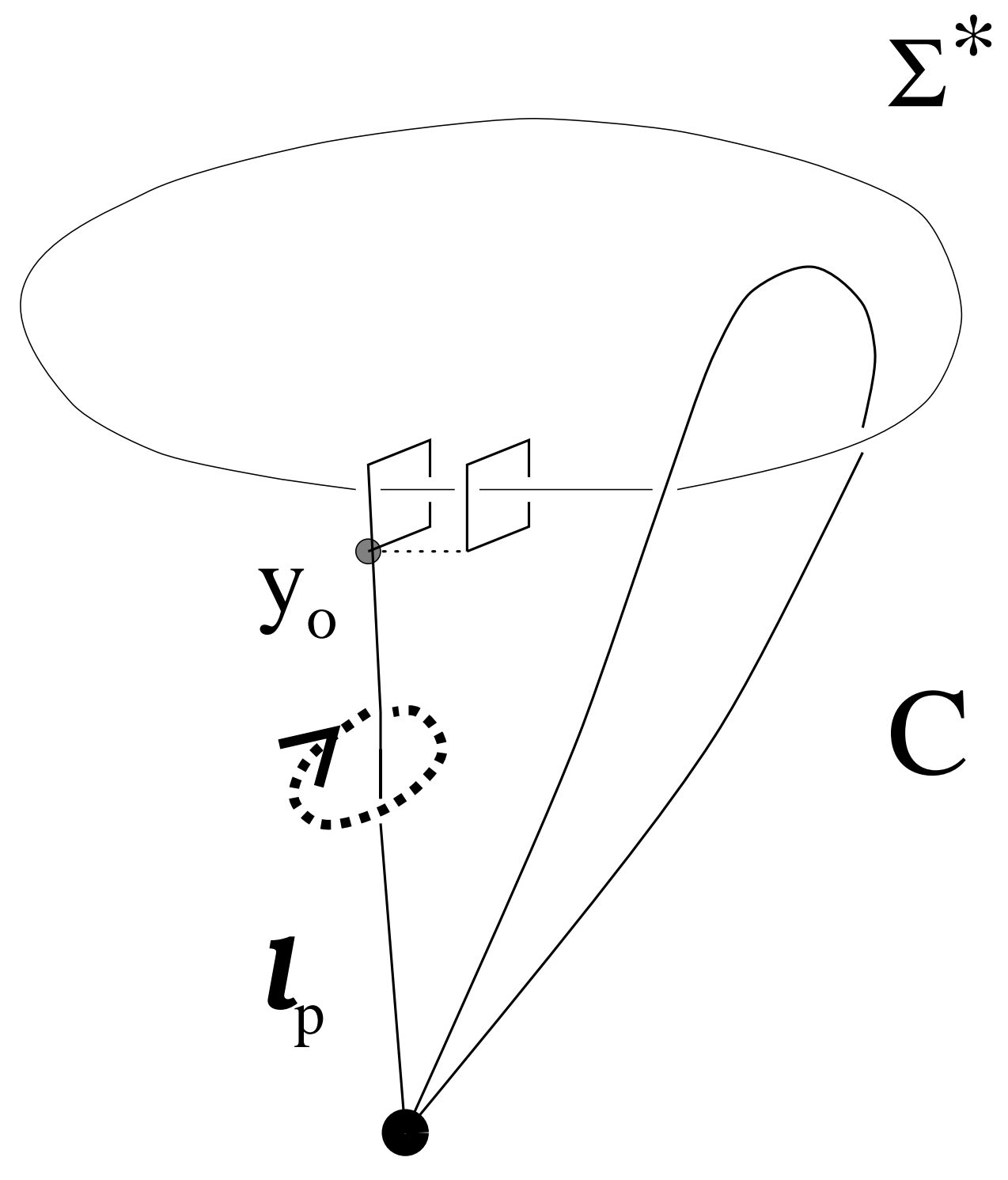

$\mathrm{X}_{0}$ 


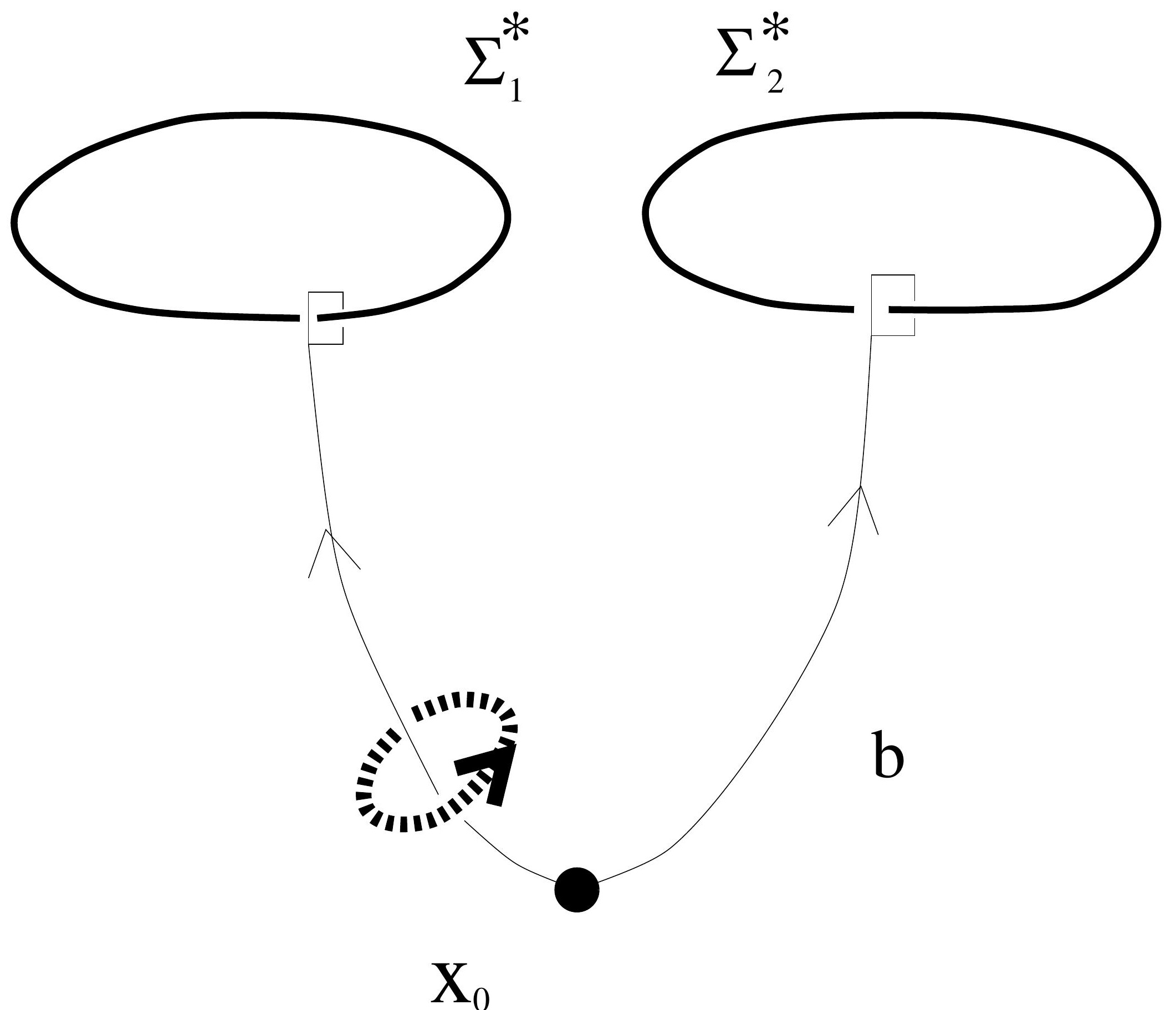




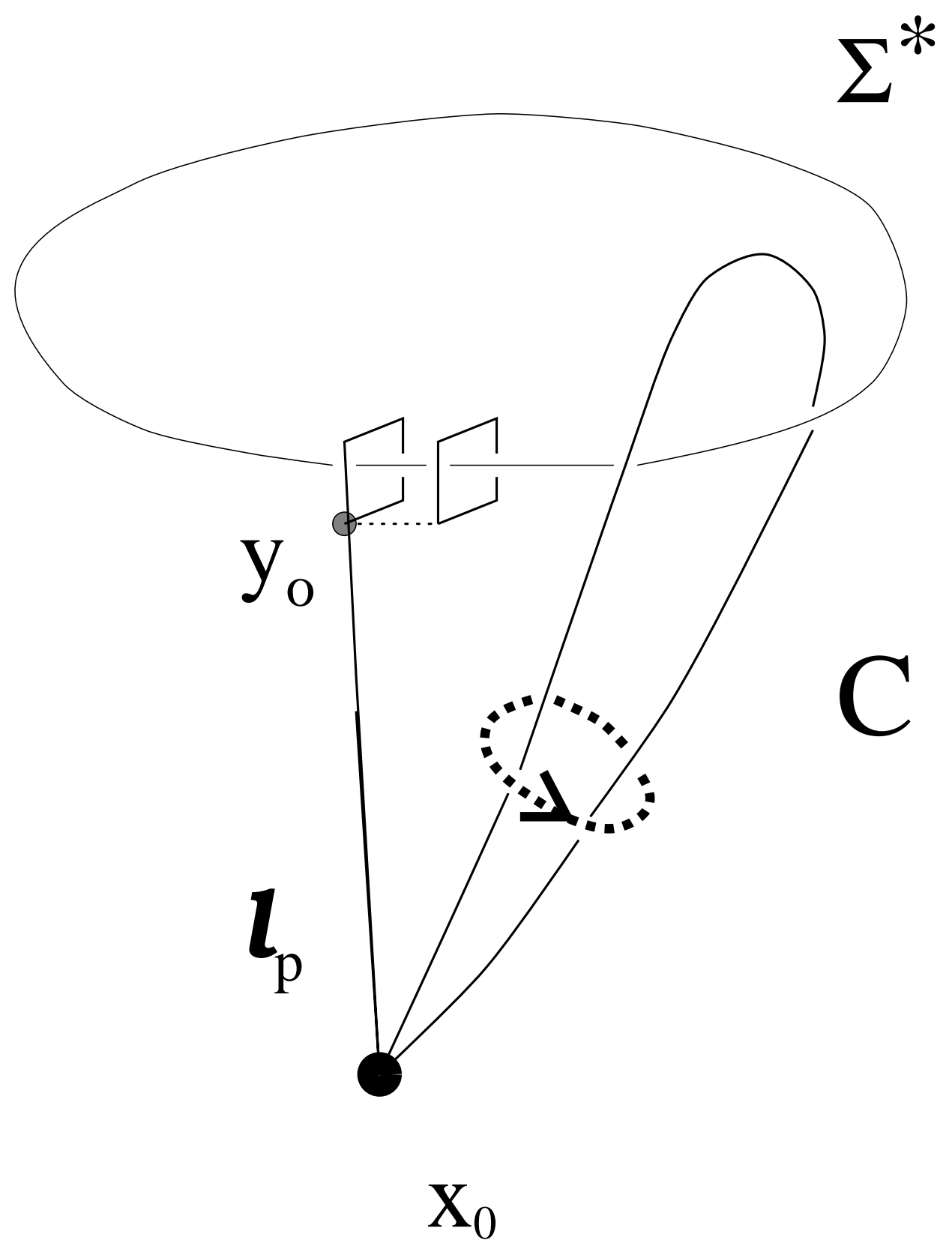




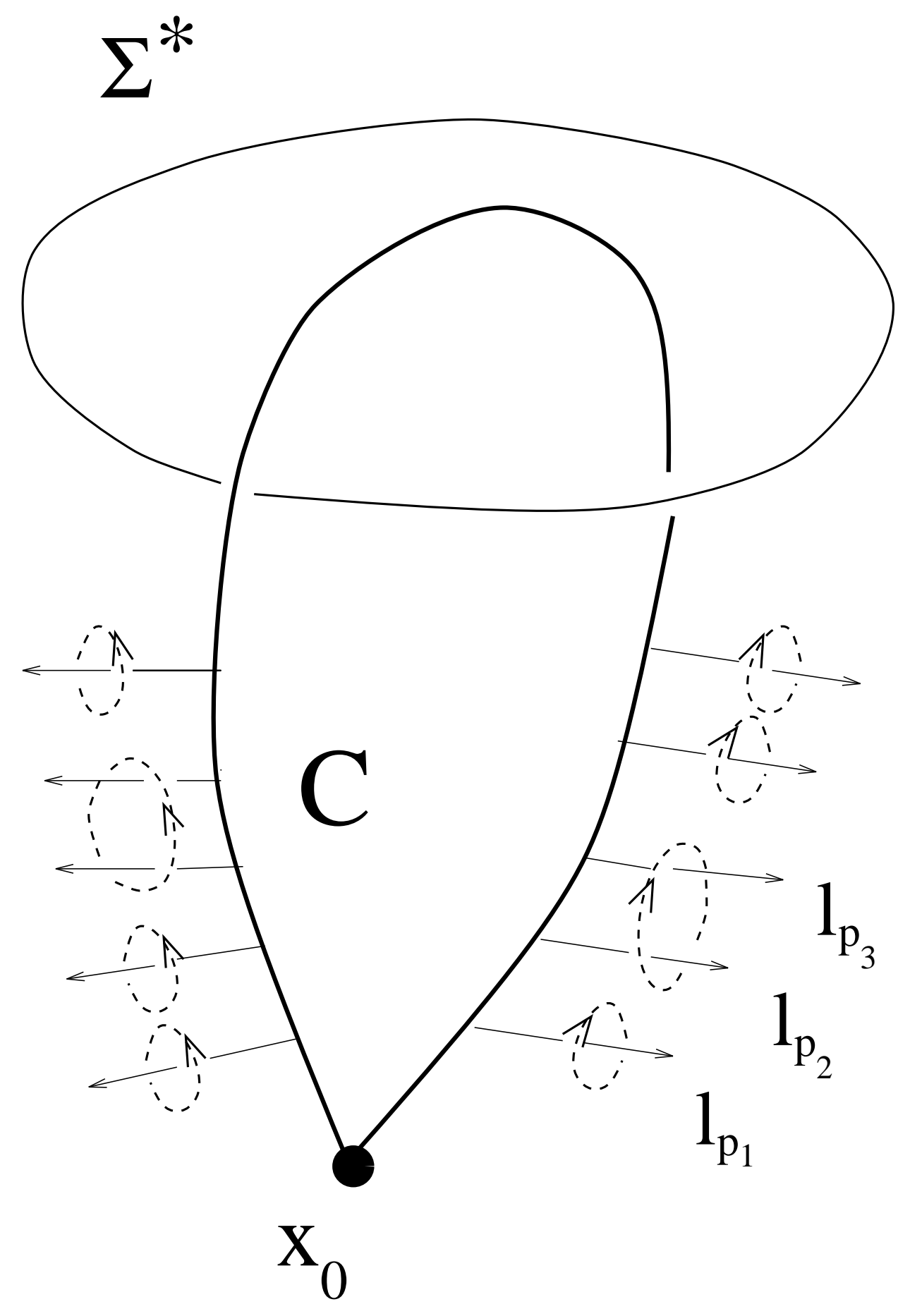




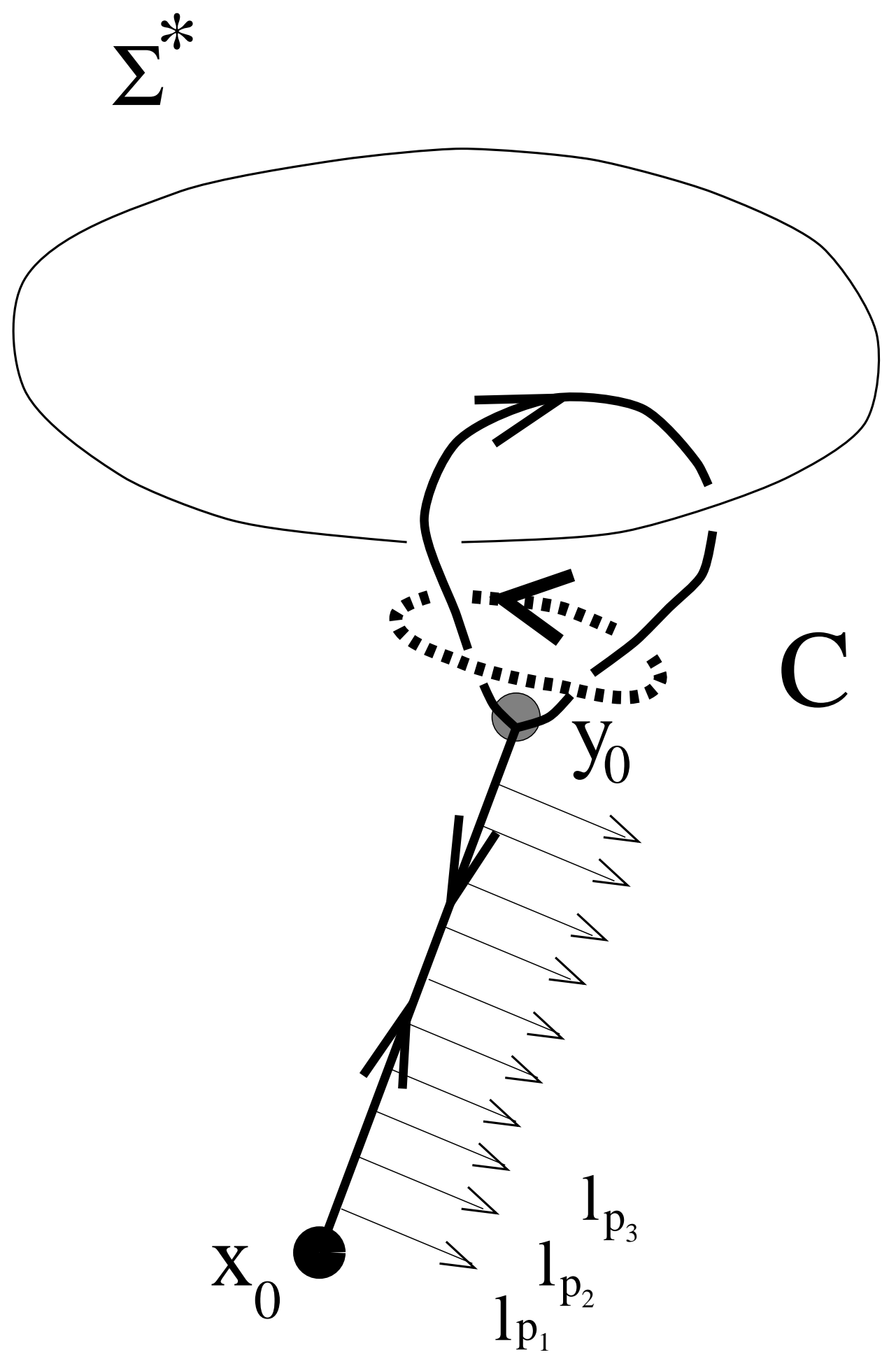




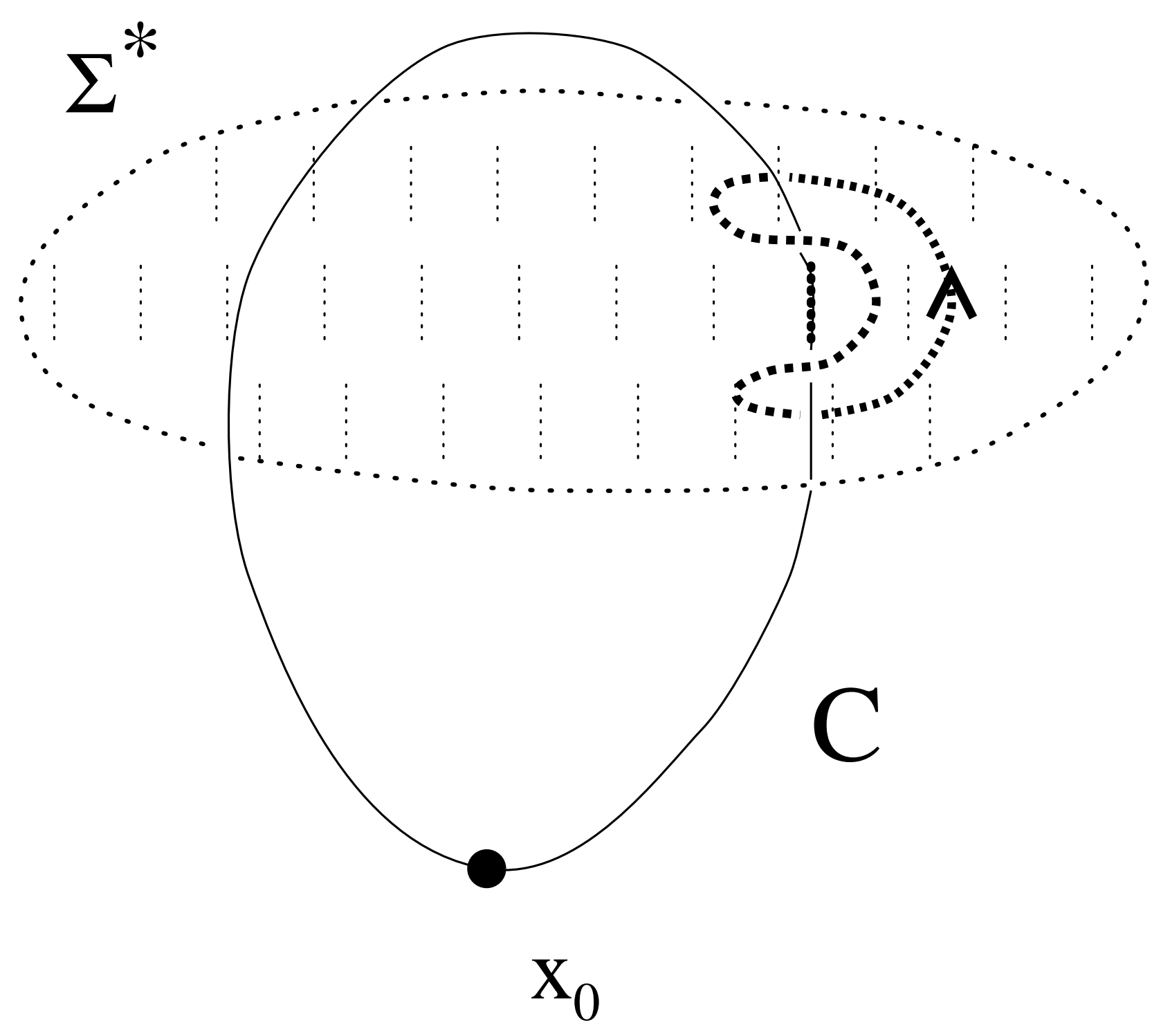




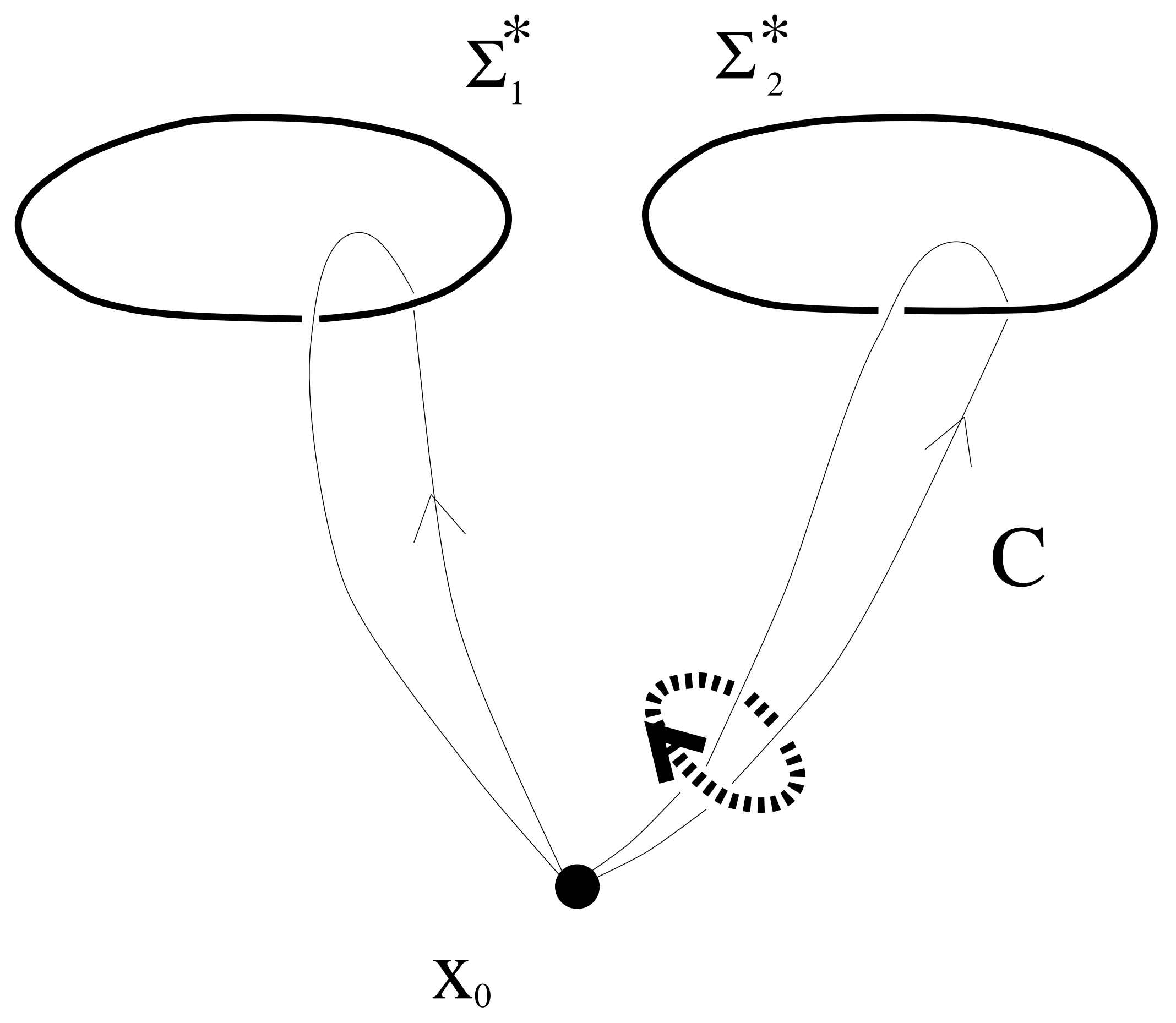




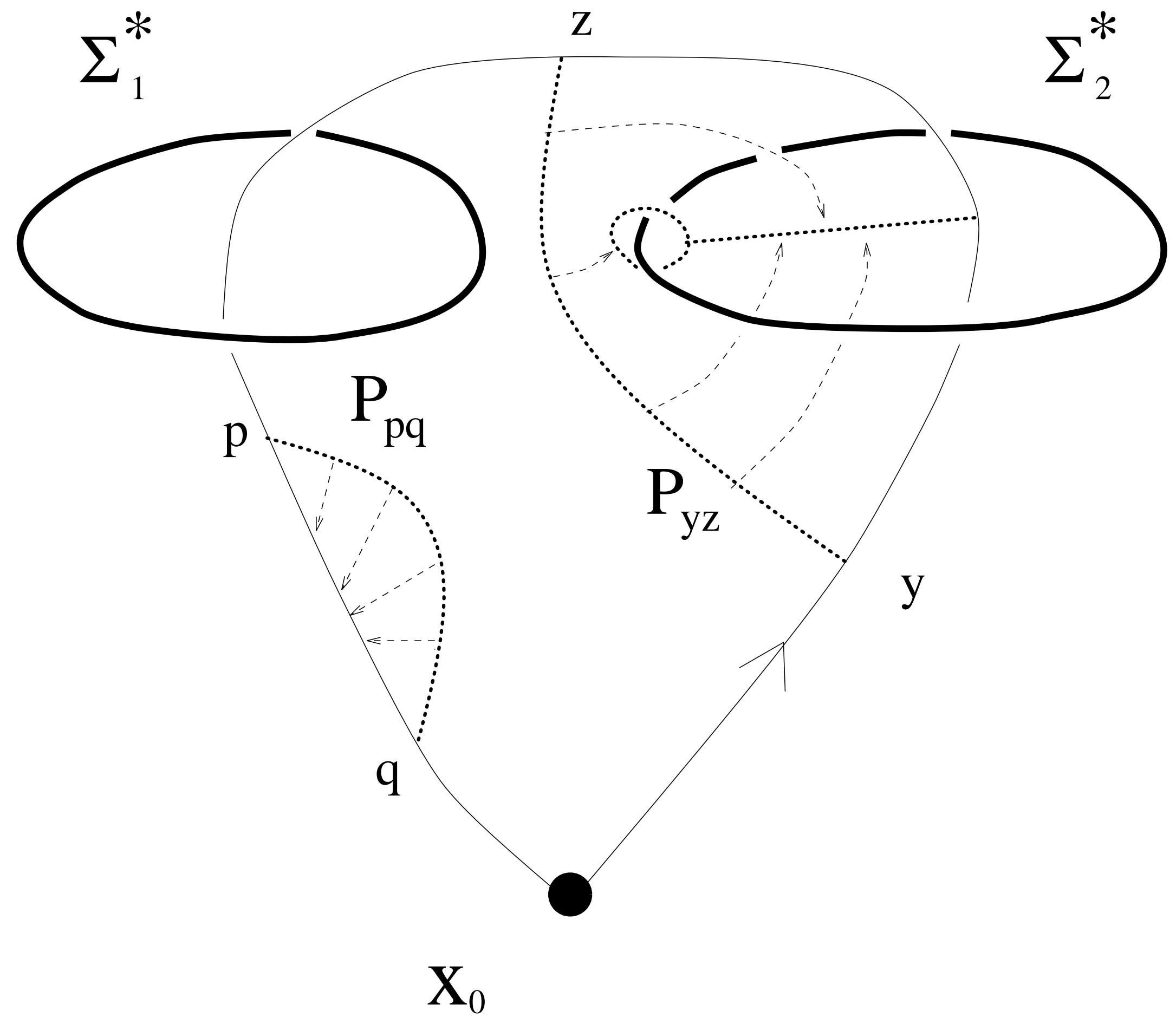




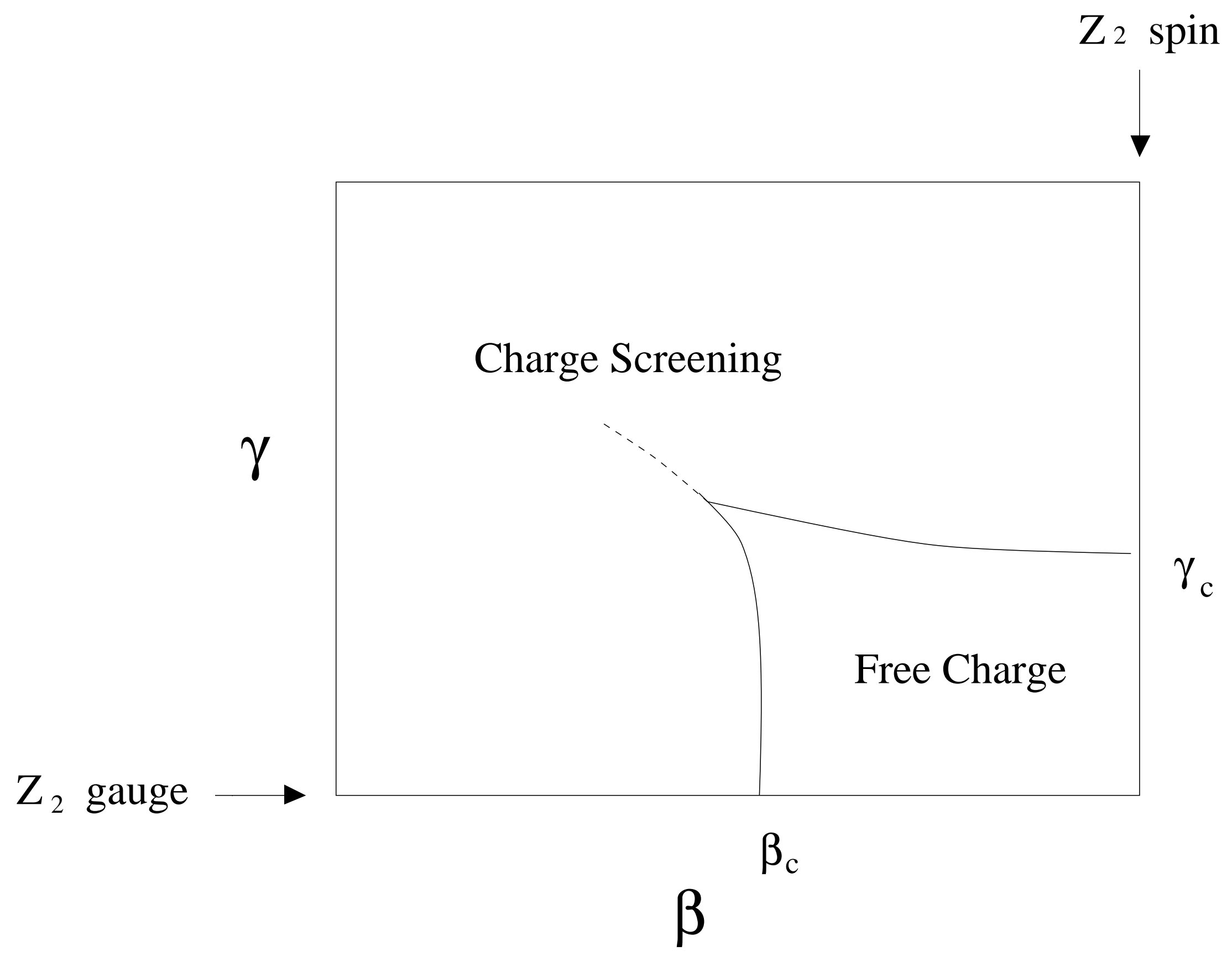




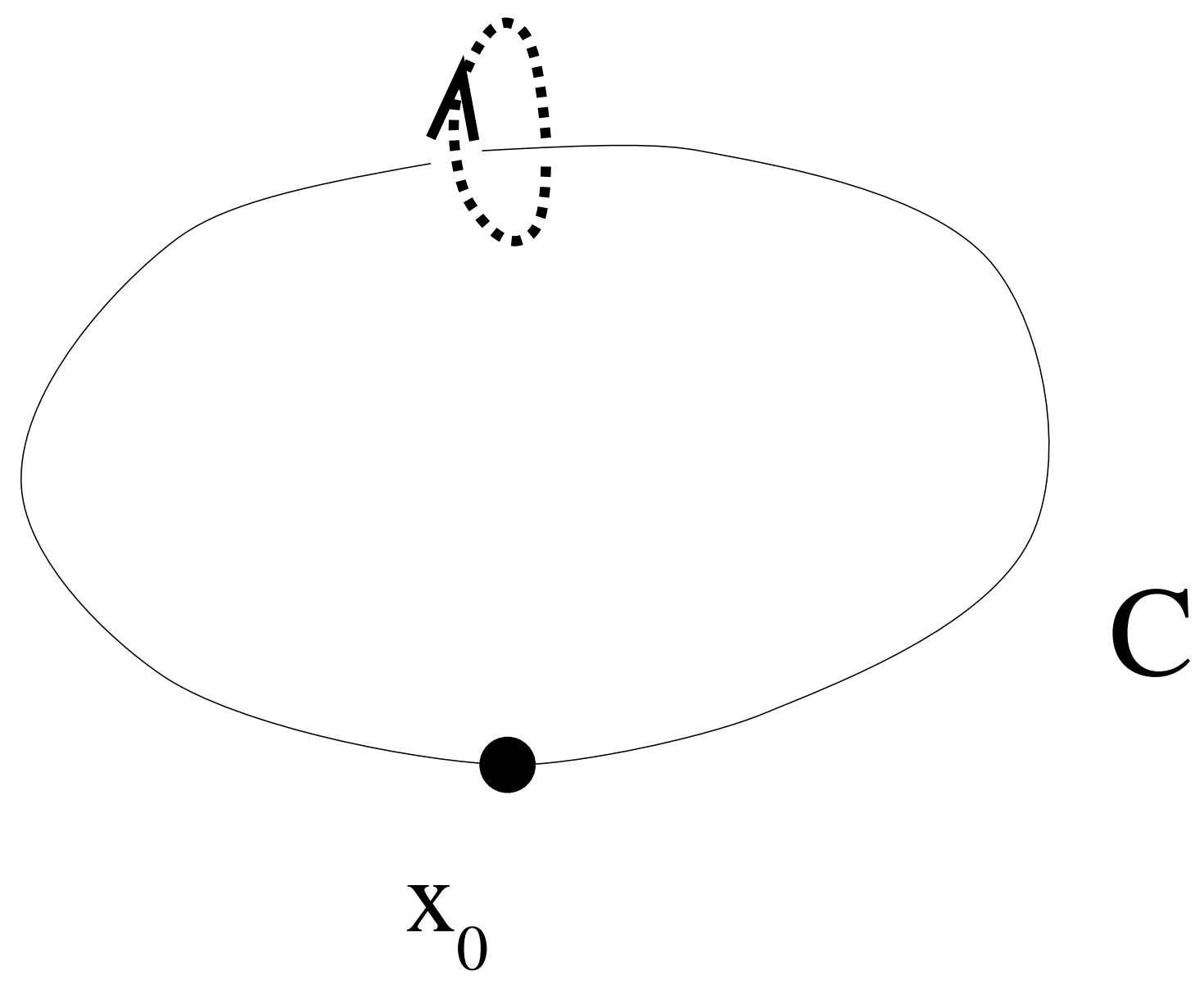

